\title{
Neutron-induced astrophysical reaction rates for translead nuclei ${ }^{\star}$
}

\author{
I. V. Panov ${ }^{1,2,3}$, I. Yu. Korneev ${ }^{3}$, T. Rauscher ${ }^{1}$, G. Martínez-Pinedo ${ }^{4,5}$, A. Kelić-Heil ${ }^{4}$, \\ N. T. Zinner ${ }^{5}$, and F.-K. Thielemann ${ }^{1}$
}

1 Department of Physics, University of Basel, Klingelbergstr. 82, 4056 Basel, Switzerland

2 Institute for Theoretical and Experimental Physics, B. Cheremushkinskaya St. 25, 117259 Moscow, Russia

e-mail: Igor.Panov@itep.ru

3 Russian Research Center Kurchatov Institute, pl. Kurchatova 1, Moscow 123182, Russia

4 GSI Helmholtz Center for Heavy Ion Research, Planckstr. 1, 64291 Darmstadt, Germany

5 Department of Physics, Harvard University, Cambridge, MA 02138, USA

Received 27 February 2009 / Accepted 12 January 2010

\section{ABSTRACT}

\begin{abstract}
Neutron-induced reaction rates, including fission and neutron capture, are calculated in the temperature range $10^{8} \leq T(\mathrm{~K}) \leq 10^{10}$ within the framework of the statistical model for targets with the atomic number $84 \leq Z \leq 118$ (from Po to Uuo) from the neutron to the proton drip-line. Four sets of rates have been calculated, utilizing - where possible - consistent nuclear data for neutron separation energies and fission barriers from Thomas-Fermi (TF), Extended Thomas-Fermi plus Strutinsky Integral (ETFSI), Finite-Range Droplet Model (FRDM) and Hartree-Fock-Bogolyubov (HFB) predictions. Tables of calculated values as well as analytic seven parameter fits in the standard REACLIB format are supplied. We also discuss the sensitivity of the rates to the input, aiming at a better understanding of the variations introduced by the nuclear input.
\end{abstract}

Key words. nuclear reactions, nucleosynthesis, abundances - supernovae: general - stars: neutron

\section{Introduction}

Investigations of nucleosynthesis processes make use of reaction networks including thousands of nuclei and tens of thousands of reactions. Most of these reactions occur far from stability and thus cannot yet be directly studied in the laboratory. In addition most of the nuclear properties including reaction rates, which are also required for the calculation of cross sections and astrophysical reaction rates, are not experimentally known either. Therefore, predictions based on theoretical models are necessary. While close to stability partial experimental information is available, relying fully on theoretical information leads to relatively large variations in computed cross sections far from stability. This is especially true for the region of fissionable nuclei, which is the focus of the present investigation.

In the past, a series of efforts were applied to calculate neutron-capture rates for r-process nucleosynthesis and other astrophysical applications (e.g., Arnould 1972; Holmes et al. 1976; Woosley et al. 1978; Sargood 1982; Thielemann et al. 1987; Cowan et al. 1991; Rauscher \& Thielemann 2000; Aikawa et al. 2005; Goriely et al. 2008, and references therein).

Fission has often been neglected in astrophysical calculations. In early applications to astrophysical nucleosynthesis, usually only one mode was considered, beta-delayed fission (Thielemann et al. 1983) or a phenomenological model of spontaneous fission (Goriely \& Clerbaux 1999; Freiburghaus et al. 1999; Cowan et al. 1999). However, it was shown recently that

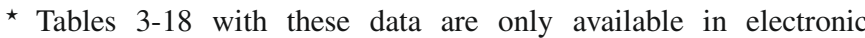
form at the CDS via anonymous ftp to cdsarc.u-strasbg.fr $(130.79 .128 .5)$ or via

http://cdsweb.u-strasbg.fr/cgi-bin/qcat?J/A+A/513/A61 neutron-induced fission is more important than beta-delayed fission in r-process nucleosynthesis (Panov \& Thielemann 2003, 2004; Martínez-Pinedo et al. 2007). Thus, the need to provide a compilation of neutron-induced fission rates is obvious. Initial investigations have been undertaken by Panov et al. (2005) and Goriely et al. (2009). Here we present extended calculations of neutron-induced fission rates for different predictions of masses and fission barriers. The present work also completes existing nuclear neutron-capture rate sets by extending the works of Rauscher \& Thielemann (2000) and Panov et al. (2005) to the region $84 \leq Z \leq 118$ in order to provide the necessary input for nucleosynthesis studies under high neutron densities. As in Panov et al. (2005), the statistical model approach of WolfensteinHauser-Feshbach (Wolfenstein 1951; Hauser \& Feshbach 1952) for compound nuclear reactions was used, but employing more recent and complete data and predictions for masses, spins, and fission barriers.

Nuclear mass and fission barrier predictions have a strong model dependence, and none of the existing models can reproduce all experimentally known data. Moreover, the fission process itself is complicated, and extended calculations for neutroninduced fission across the nuclear chart have to be done carefully. Here, we aim to provide rates for studying the endpoint of the r-process and the possible production of super-heavy elements. By comparing rates obtained with different choices of mass and fission barrier predictions we attempt to give a measure of the involved variations. Astrophysical models, providing the nucleosynthesis conditions, bear large variations in themselves. This is especially true for the r-process, for which the astrophysical site is still unknown despite decades of study. For a realistic and exhaustive exploration of synthesis conditions, simulations 
do not only have to vary astrophysical parameters, but also have to include a variation range of involved reaction rates given by different mass and fission barrier models.

Our paper is structured as follows. In Sect. 2 we briefly describe the statistical model used in the calculations as well as the nuclear input data and give a comparison of cross sections or rates for a number of experimentally known nuclei with existing experimental information and other theoretical models. These methods are then applied to supplement the rate sets of Rauscher \& Thielemann (2000) of $(n, \gamma)$-rates for chemical elements with $Z>83$ and predict neutron-induced fission cross sections and rates (where available in comparison to experiments). Section 3 presents these results and shows the sensitivity with respect to mass models and fission barriers employed. Rate fits for utilization in astrophysical calculations are discussed in Sect. 4. In Sect. 5 we give a brief discussion and some examples of the mass distribution of fission fragments, which will be provided in an extended way in a forthcoming paper. The final Sect. 6 contains conclusions and a summary. The explanation of the tables and their structure are given in Appendix A. The complete tables of reaction rates and their fits are found at CDS in electronic form.

\section{The statistical model and nuclear data input}

As in previous approaches (e.g., Thielemann et al. 1987; Cowan et al. 1991; Rauscher \& Thielemann 2000) we have applied the statistical Wolfenstein-Hauser-Feshbach formalism (Wolfenstein 1951; Hauser \& Feshbach 1952) for the calculation of neutron-induced cross sections and reaction rates. In addition to $(n, \gamma)$-reactions the fission channel was also included as outlined in Thielemann et al. (1983, 1989), Cowan et al. (1991), Panov et al. (2005). The statistical model is applicable for astrophysical rate calculations as long as there is a sufficiently high density of excited states in the compound nucleus at the relevant bombarding energy, which is the case for most heavy nuclei. However, at shell closures and with decreasing neutron separation energies, level densities at the astrophysically relevant compound formation energy in neutron-induced reactions become too small for the application of the model, as shown by Rauscher et al. (1997). In those cases, single resonances and contributions from the direct reaction mechanism have to be taken into account (Rauscher et al. 1998; Goriely \& Khan 2002). This underlines on the one hand that reliable mass predictions for the separation energies are absolutely necessary for r-process applications far from stability, and on the other hand that the prediction of spectroscopic properties for resonant and direct capture awaits improvement as well. Here we calculate neutron-induced rates still based purely on the statistical model to provide a full set of rates for extended r-process calculations and the possible formation of superheavy nuclei for a variety of different sets of mass models and fission barrier predictions. The influence of the direct reaction mechanism on the rates far from stability needs to be explored separately in a future study.

We outlined the general treatment within the statistical model for applications, the cross section for a neutron-induced reaction $i^{0}(n$, out) ("out" standing for gamma-emission or fission) from the target ground state $i^{0}$ with center of mass energy $E_{\text {in }}$ and reduced mass $\mu_{\text {in }}$ given by

$$
\begin{aligned}
\sigma_{(n, \text { out })}^{0}= & \frac{\pi \hbar^{2}}{\left(2 \mu_{\text {in }} E_{\text {in }}\right)} \times \frac{1}{\left(2 J_{i}^{0}+1\right) \cdot\left(2 J_{n}+1\right)} \\
& \times \sum_{J, \pi}(2 J+1) \frac{T_{n}^{0}\left(E, J^{\pi}, E_{i}^{0}, J_{i}^{0}, \pi_{i}^{0}\right) T_{\text {out }}\left(E, J^{\pi}\right)}{T_{\text {tot }}\left(E, J^{\pi}\right)} .
\end{aligned}
$$

The total transmission coefficient $T_{\text {tot }}=\sum_{v, o} T_{o}^{v}$ describes the transmission into all possible bound and unbound states $v$ in all energetically accessible exit channels $o$ (including the entrance channel $i$ ). The fission transmission coefficient $T_{f}\left(E, J^{\pi}\right)$ includes the sum over all possible final states and is evaluated as discussed in Bjornholm \& Lynn (1980), Cowan et al. (1991), Panov et al. (2005) and is related to the fission probability $P_{f}\left(E, J^{\pi}\right)=T_{f}\left(E, J^{\pi}\right) / T_{\text {tot }}\left(E, J^{\pi}\right)$ considered in the papers cited above. Since the work of Strutinsky (1967) fission has been generally described within the framework of double-humped fission barriers. When making use of a double-humped fission barrier, the fission transmission coefficients can be calculated in the limit of complete damping, which averages over transmission resonances, assuming that levels in the second minimum between the first and second barrier $E_{A}$ and $E_{B}$ are equally spaced. If detailed information concerning the level structure in the second well is missing, this method gives the best results. $P_{f}$ further requires the transmission coefficients through the first and second barrier $T_{A}$ and $T_{B}$, which are evaluated by an integral over the first and second barrier potential, weighted with the level density at the appropriate energy and corresponding deformation. The individual barriers can be approximated by individual (inverted parabola) Hill-Wheeler barrier shapes. The level densities $\rho_{A}$ and $\rho_{B}$ show an enhancement over the level densities at ground state deformation, and it is important to include proper symmetry classes in the calculations at corresponding saddle points. In the absence of detailed information, standard factors of four (for the axially asymmetric/mass symmetric barrier) and two (axially symmetric/mass asymmetric) over the ground state level density were applied (Bjornholm \& Lynn 1980). Whenever possible, experimentally known fission barriers were used, taken from Smirenkin (1993), the compilation of Mamdouh et al. (1998), and the database of Belgya et al. (2006). The other transmission coefficients were calculated as in Rauscher \& Thielemann (2000), utilizing up to 19 experimentally known excited states (if available). The data were taken from Firestone \& Shirley (1996), up to the first level for which the spin assignment is unknown. Ground state spins and parities are known for many unstable nuclei. Far off stability, ground state spins and parities were taken from Möller et al. (1997) when experimental values were unavailable. Above the last known state, the nuclear level density of Rauscher et al. (1997) was used. This method is based on the back shifted Fermi-gas approach, where the level density parameter $a$ and back shift $\delta$ are obtained globally from the appropriate mass model employed.

In Fig. 1 we compare our predictions for neutron-induced fission cross sections of some $\mathrm{U}, \mathrm{Np}$ and $\mathrm{Pu}$ isotopes with evaluated neutron data from JENDL-3.3 (Nakagawa et al. 2005; Soppera et al. 2008). (The accuracy of the evaluated data is usually not declared. In the region of interest, $0.01<T_{9}<\mathrm{MeV}$, the accuracy of up-to-date measurements for plutonium isotopes by Tovesson et al. 2009 varies from $2 \%$ to $15 \%$, and for our plots is not bigger than the plot signs. The detailed experimental information can be found in the experimental nuclear reaction data library 2009.) Experimental masses and fission barriers or masses and fission barriers were employed from different mass models: ETFSI (Aboussir et al. 1995; Mamdouh et al. 1998), TF (Myers \& Swiatecki 1996, 1999), HFB-14 (Goriely et al. 2009) and the older liquid drop predictions by Howard \& Möller (1980), here shown as Panov et al. 2005). It can be seen that when using experimental fission barriers, the agreement with experimental $(n, f)$ cross sections is within the typical factor of two to three known for statistical model calculations. It can also be seen that different barrier predictions can lead to large 
I. V. Panov et al.: Neutron-induced rates...
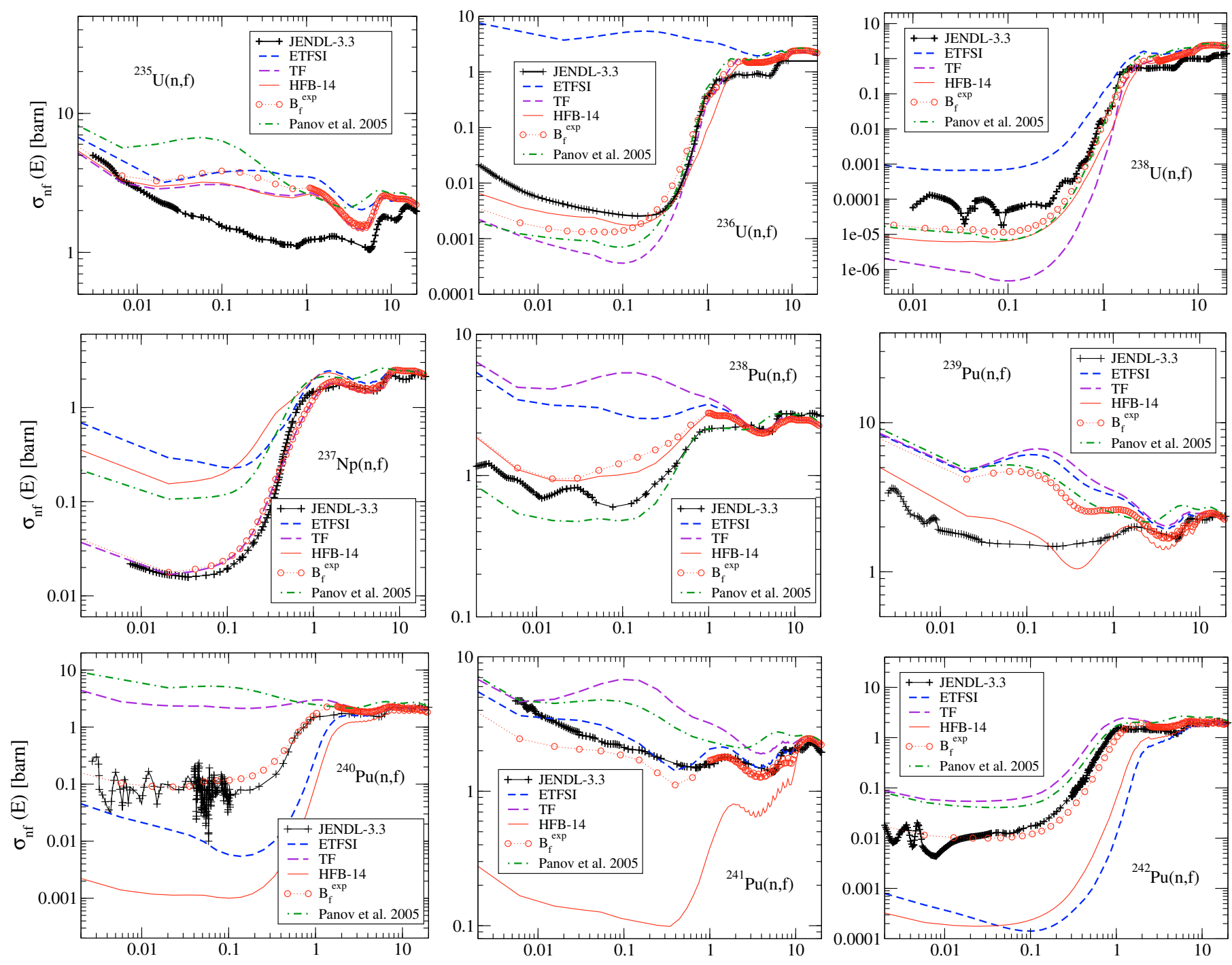

$\mathrm{E}, \mathrm{MeV}$

$\mathrm{E}, \mathrm{MeV}$

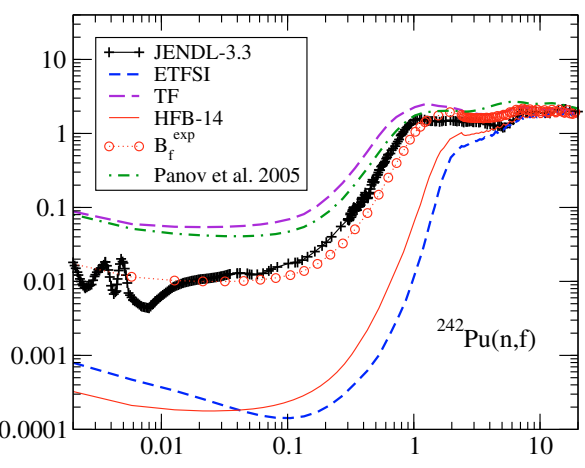

Fig. 1. Present predictions of energy-dependent $(n, f)$ cross sections $\sigma_{n f}(E)$ for some target nuclei of $\mathrm{U}, \mathrm{Np}$ and Pu calculated in the framework of different mass and fission barrier predictions (ETFSI, TF, HFB-14) and experimental data, marked $B_{\mathrm{f}}^{\text {exp }}$ as well. Experimentally measured crosssections were used after JENDL-3.3 (Nakagawa et al. 2005), averaged by the code JANIS Soppera et al. (2008), displayed by a black line. All the predictions are given for a ground-state population. Our previous results (Panov et al. 2005) are shown as well.

variations, which will clearly remain for predictions far from stability where no experimental information is available. These results can also be compared to a recent investigation by the Reference Input Parameter Library (RIPL) community (Goriely et al. 2009), making use of the code TALYS and HFB-14 fission barriers plus nuclear level densities obtained from a combinatorial approach based on single particle spectra from the corresponding Hartree-Fock-Bogolyubov calculations. The fission barriers in (Goriely et al. 2009) were employed following the fission path via a Wentzel-Kramers-Brillouin phase integral, which should be superior to a Hill-Wheeler inverted parabola treatment. When examining their Fig. 8, which is based on pure predictions, it can be recognized that our results with HFB-14 fission barriers employed via a double-humped fission barrier approach are similar or even closer to the experiment than the results given therein. As we make use of the same barriers, we relate the difference mainly to the different level density predictions (here a back-shifted Fermi gas with parameters obtained from a global mass model, there a combinatorial approach to single particle spectra from a microscopic HFB mass model). We draw the same conclusions from the comparison with their Fig. 9 and our calculations with experimental barriers. While microscopic investigations should be in principle more advanced, the back-shifted Fermi gas approach based on global mass models seems still more robust in its predictive power. While renormalizations of fission paths and level densities can improve this picture (see their Figs. 10-12), this is only possible if experimental data are available. As our investigations are meant for astrophysical applications far from stability, where no experimental information is available, we come to the conclusion that our approach is well suited for this endeavor. It does, however, depend on the quality of mass and fission barrier predictions, and this will be the focus of the present work.

In astrophysical plasmas, reactions also occur on thermally excited target states. The stellar cross section $\sigma^{*}$ can then be defined as a sum of the cross sections $\sigma^{x}$ for those excited 

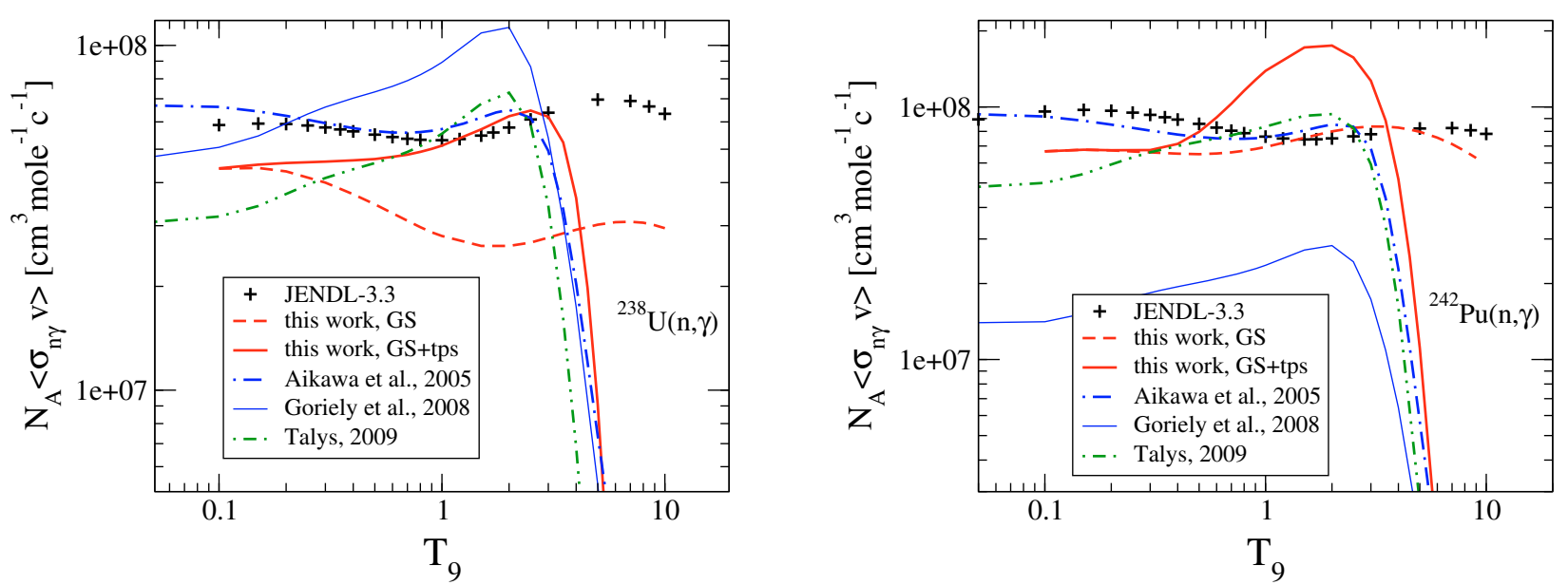

Fig. 2. Comparison of $(n, \gamma)$-rates (integrated over Maxwell-Boltzmann distributions of targets and projectiles for the displayed temperatures) from present calculations and other existing predictions for the target nuclei ${ }^{238} \mathrm{U}$ (left) and ${ }^{242} \mathrm{Pu}($ right $)$ with experiment. The symbols are chosen as follows: Aikawa et al. (2005) (blue dash-dot line), Goriely et al. (2008) (thin blue line), JENDL-3.3 (Nakagawa et al. 2005) (crosses), new Talysbased (Goriely et al. 2009) predictions (green dash-dot line). Our calculations are shown for reaction rates including only the ground state (GS) or a thermally populated target (GS+tps). Only the GS rates can be compared to experimental data. The results of Aikawa et al. (2005); Goriely et al. (2008) correspond to GS + tps conditions.
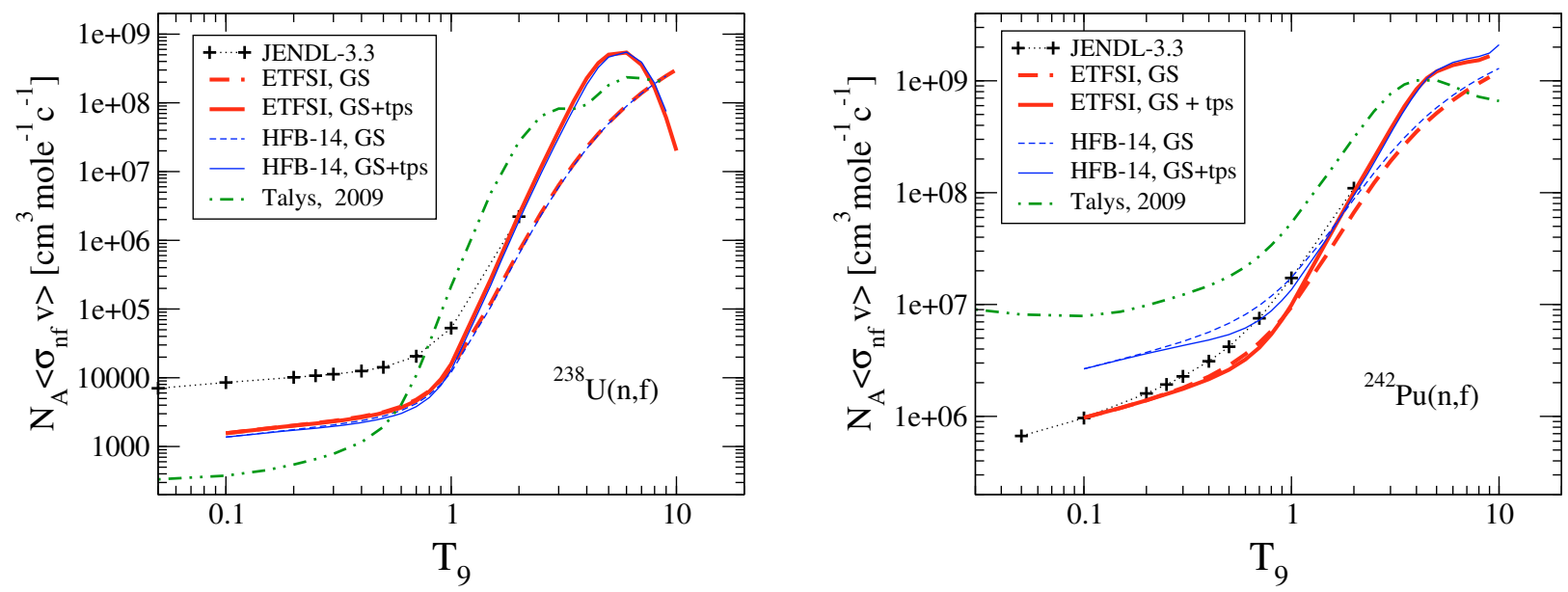

Fig. 3. Comparison of $(n, f)$-rates (integrated over Maxwell-Boltzmann distributions of targets and projectiles for the displayed temperatures) from present calculations and other existing predictions for the target nuclei ${ }^{238} \mathrm{U}(l e f t)$ and ${ }^{242} \mathrm{Pu}($ right $)$ with the experiment. The symbols are chosen as follows: Goriely et al. (2009) (green two-dot-dash line), JENDL-3.3 (Nakagawa et al. 2005) (crosses). Our calculations are shown for reaction rates including only the ground state (GS) or a thermally populated target (GS+tps) for different nuclear data predictions (HFB or ETFSI). The results of Goriely et al. (2009) correspond to GS + tps conditions.

states $x$ with excitation energy $E_{x}$ and spin $J_{x}$, weighted by the Boltzmann excitation probability

$\sigma^{*}=\frac{\sum_{x}\left(2 J_{x}+1\right) \sigma^{x} \mathrm{e}^{-\frac{E_{x}}{k T}}}{\sum_{x}\left(2 J_{x}+1\right) \mathrm{e}^{-\frac{E_{x}}{k T}}}$.

The $\sigma^{x}$ were calculated in the same way as shown in Eq. (1) for the ground state, i.e. for $x=0$. Only the stellar cross sections can be used to compute the appropriate astrophysical reaction rates. The reaction rate for a specific reaction at a given stellar temperature $T$ was then determined by folding the stellar reaction cross section $\sigma^{*}(E)$ with a Maxwell-Boltzmann distribution of relative velocities between projectiles and targets (Fowler 1974):

$$
\left\langle\sigma^{*} v\right\rangle=\langle\sigma v\rangle^{*}=\left(\frac{8}{\pi \mu}\right)^{1 / 2} \frac{1}{(k T)^{3 / 2}} \int_{0}^{\infty} \sigma^{*}(E) E \exp \left(-\frac{E}{k T}\right) \mathrm{d} E
$$

Figures 2 and 3 show a typical comparison of present neutron capture rate calculations of ${ }^{238} \mathrm{U}$ and ${ }^{242} \mathrm{Pu}$ to experimentally based rates from JENDL-3.3 (Nakagawa et al. 2005) and the predictions of Aikawa et al. (2005); Goriely et al. (2008, 2009). The agreement between rate predictions and data for other nuclei in this mass range are of the same order. Note that these predictions along the valley of stability are based on experimental masses, which leads to an average agreement with experimental cross sections within a factor of 1.5. The expected variation will be larger far from stability where theoretical mass and barrier predictions have to be utilized.

\section{Neutron-induced fission rates for a variety of mass models}

Early r-process calculations (Thielemann et al. 1983), which included fission, made use of the mass predictions by Hilf et al. (1976) and the fission barriers of a macroscopic-microscopic model by Howard \& Möller (1980). For many years different authors used the fission barriers from Howard \& Möller (1980), as they were the only complete set of barriers available. More 

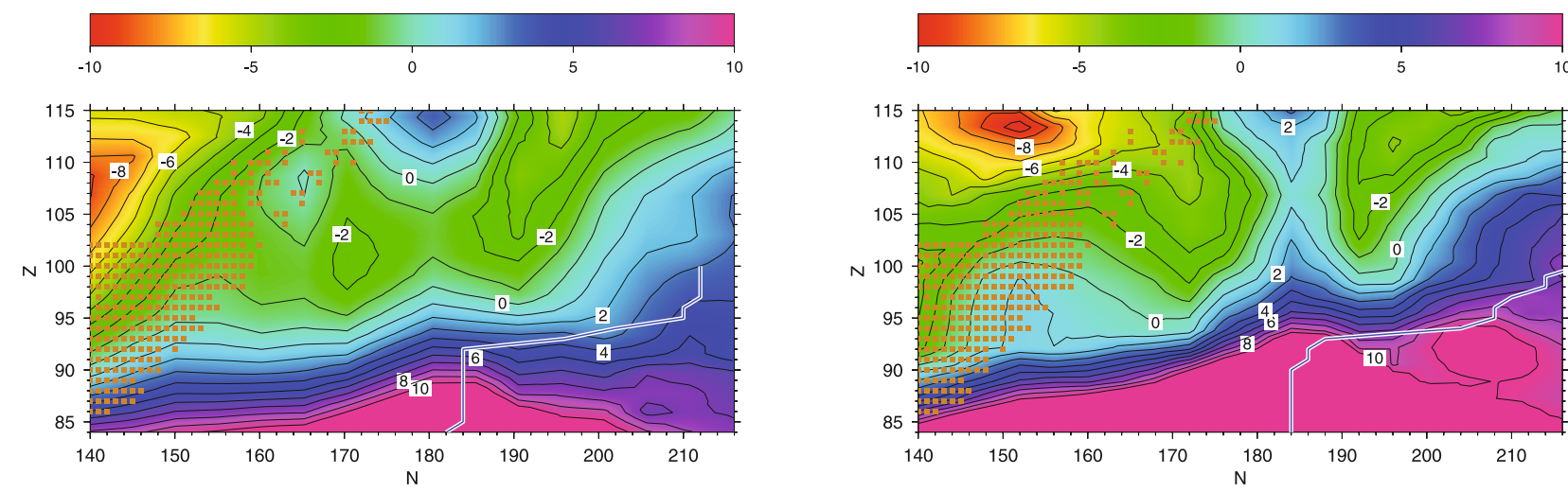

Fig. 4. Contour plot of the quantity $B_{f}-S_{n}$ (in MeV) for two choices of models for masses and fission barriers: FRDM masses plus TF fission barriers (left) and ETFSI-predictions (right). Also indicated are known superheavy nuclei (boxes) and the drip-line (line), where $B_{f}-S_{n}$ determines whether the r-process continues towards heavier nuclei or fission cycling to lighter fission products occurs.

recently, renewed interest (and increased computing power) spurred a number of new calculations of large sets of barrier predictions within various models, resulting on "average" in higher values of fission barriers than predicted by Howard \& Möller (1980). For a consistent treatment of nucleosynthesis, fission rates should be calculated with the neutron separation energies, reaction Q-values and fission barrier heights derived from the same mass model (see the discussion in Cowan et al. 1991; Rauscher et al. 1994).

As explained in Sect. 2, different mass- and fission barrier predictions were utilized to test the sensitivity stemming from different underlying models. The models used are: masses taken from the Finite Range Droplet Model (FRDM) by Möller et al. (1995), the Extended Thomas-Fermi with Strutinsky Integral (ETFSI) model by Aboussir et al. (1995), and the Thomas-Fermi (TF) model of Myers \& Swiatecki (1996); fission barriers are taken from the ETFSI (Mamdouh et al. 2001) and TF (Myers \& Swiatecki 1999) models. It should be emphasized that the ETFSI masses employed here (http://www-astro.ulb. ac.be/Nucdata/Masses/etfsi2-plain) are based on the force SkSC18 (Goriely 2000), while the ETFSI fission barriers (http://www-astro.ulb.ac.be/Nucdata/Fisbar/ fisbar1) were obtained with the force SkSC4 (Mamdouh et al. 1998). Thus, it is difficult to perform fully consistent calculations, and the quality of barrier heights is not really known especially in the region far from experimentally known nuclei, where the r-process proceeds. For this reason the choice of two different sets of fission barriers, TF and the ETFSI, permits to test the sensitivity range. When utilized together with mass predictions from the same models (see however the remarks in the previous paragraph), a reasonably consistent treatment is possible. In addition, we also chose to explore a combination of TF fission barriers and FRDM nuclear mass predictions and to gauge the resulting effect. The latter are close to the TF predictions, containing also the same shell corrections (Myers \& Swiatecki 1996) and have already been used in some astrophysical rate calculations for nuclei with $Z<84$.

Figure 4 shows the quantity of primary importance, $B_{f}-S_{n}$, for the calculation of neutron-induced fission cross sections, based on the two model sets FRDM+TF and ETFSI. $B_{f}-S_{n}$ indicates the regions of nuclei where neutron induced fission rates can be high enough $\left(B_{f}-S_{n}<0\right)$ and are important for the rprocess nucleosynthesis (see also the discussion of Fig. 6). We see that both sets display a quite different behavior and note that $B_{f}-S_{n}$ is generally larger for ETFSI than FRDM+TF.
Our fission cross section calculations were performed within the framework of a double-hump fission barrier (permitting the existence of a double or single hump barrier). The heights of both barriers $f$ were predicted in the calculations of Howard \& Möller (1980) (HM) and Mamdouh et al. (2001). However, the TF model predicts only one of the fission barriers. In order to employ this model in our calculations we assumed that the predicted fission barrier is the higher of both barriers and derived the lower one as described below and in Panov et al. (2005). In order to derive the height of the lower barrier, we compared two methods: (i) keeping the difference in height of the first and second barrier of the original HM barriers and (ii) keeping the same height ratio, i.e. relative height, of the barriers. The resulting cross sections were not very different because the heights of the lower barriers calculated in these two ways differ only by a few percent for the majority of cases. Only in a small number of cases the cross sections differ by more than $10 \%$ (but the largest deviations do not exceed a factor of two). For the rates shown here we chose to use the difference in height of the first and second barrier of HM to predict the lower barrier for the TF approach. In this manner, the new fission rate calculations were extended to the region above charge $Z=100$, whereas Howard $\&$ Möller (1980) presented results only for $Z \leq 100$. This allows us to perform r-process calculations in extremely neutron-rich environments as well as to study superheavy element production in rapid neutron capture nucleosynthesis processes.

An extended comparison to evaluated neutron-induced fission cross sections, based on evaluated data (Nakagawa et al. 2005) for the trans-lead region is shown in Fig. 5. The left panel displays the ratio of predicted to evaluated cross section when using experimentally known fission barriers (circles). The agreement of the calculated rates based on the experimental values of fission barriers is quite good with the majority of ratios, and is within factors of two to three. Some outliers of up to a factor of ten are observed in a few cases, but the experimental accuracy of the barrier determination is not known for these cases. The middle panel shows the difference between the calculated ones when the HFB mass and fission barrier predictions were used: in this work (triangles down) and by Talys (crosses). Existing difference in two Hauser-Feshbach calculations can have emerged from the differences in fission barriers values (due to renormalization by Goriely et al. 2009), different level density used etc. In the right panel of Fig. 5 the calculated cross sections made use of mass and fission barrier predictions from TF and ETFSI as well as new predictions by Möller et al. (2009). 

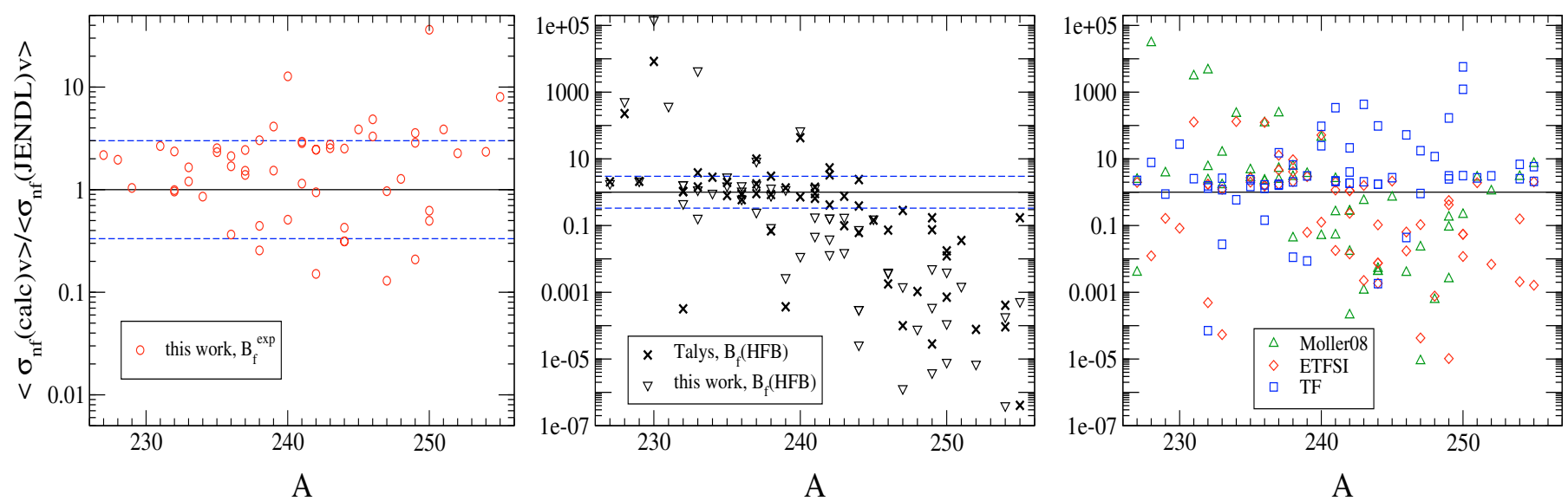

Fig. 5. Calculated maxwellian averaged neutron-induced fission cross sections in comparison to evaluated experimental fission cross sections at $30 \mathrm{keV}$. Left panel: experimental fission barriers were utilized in the calculations (circles) of maxwellian averaged cross-sections in comparison to experimental values for 58 isotopes from Th to Fm taken from JENDL-3.3 (Nakagawa et al. 2005); central panel: the same ratio of calculated and evaluated experimental values, but employing theoretical fission barrier predictions from different sources: the Extended Thomas-Fermi model by Mamdouh et al. (2001) (diamonds), the Thomas-Fermi by Myers \& Swiatecki (1999) (squares), and recent predictions from Möller et al. (2009) (green triangles up). When not experimentally known, consistent nuclear masses were utilized from the corresponding model predictions. Right panel: comparison of maxwellian averaged cross-sections from our calculations (triangles) and from the Talys (Goriely et al. 2009) calculations (crosses), both utilizing HFB predictions (with BSk14 Skyrme force) of masses and fission barriers.

Table 1. Neutron separation energy $S_{n}$ and fission barrier predictions for nucleus ${ }^{262} \mathrm{U}$ formed after neutron capture by ${ }^{261} \mathrm{U}$.

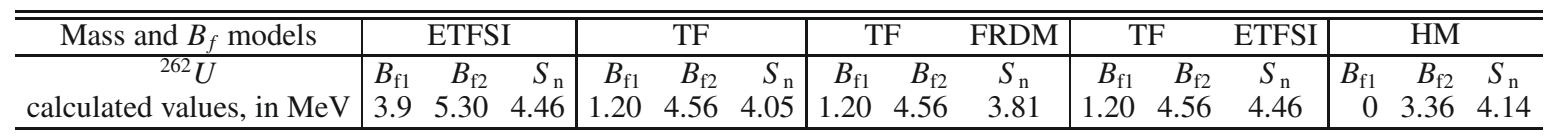

Contrary to the comparison with the left panel (circles), when using only experimental barriers we found that the difference between calculated cross sections and measured ones can be many orders of magnitude (up to a factor $10^{4}$ ). It is interesting to note that a weak systematic can be seen. Cross sections calculated with ETFSI as well as HFB fission barrier predictions show a large scatter above and below the measured values for the lighter end of the plotted mass range, whereas they tend to underpredict the fission cross sections for heavy masses. The calculations using the recent barriers by Möller et al. (2009) seem to show a similar behavior with a smaller scatter, but they are currently available only for a more limited number of nuclei. The results obtained with the TF barriers exhibit a different pattern, the predicted cross sections agree well for the lighter mass nuclei, but seem to be systematically too large for the heavier ones.

The above comparison underlines the considerable variations still inherent in fission barrier predictions. However, we suggest that by comparing TF with ETFSI (and with Möller et al. 2009) predictions, the relevant variation range can be estimated. Theoretical cross sections depend strongly on the fission barriers, and a high accuracy for their values is required. Because of the impact of the fission barrier uncertainties, nucleosynthesis studies at present should explore a variety of barrier sets, while waiting for further measurements and improved predictions. For this reason we compute and compare below rates for different sets of nuclear properties and also provide tables and fits of the rates for all these cases.

Figure 6 shows fission cross sections (left panel) and rates (right panel) calculated by combining different sets for predictions of masses and fission barriers. The arrows in the left plot show the difference between the fission barrier height and the neutron separation energy $B_{f}-S_{n}$ given by predictions of the
TF model (red arrow at the top of the left panel) and the ETFSI model (dashed arrow at the bottom). The exact values can be found in Table 1. The cross sections (and thus the rates) depend essentially on the available energy $B_{f}-S_{n}$ in the fission channel, minor dependencies on fission barriers heights and neutron separation energies, individually, are due to the competition with the $(n, \gamma)$-channel.

It can be seen from Fig. 6 that the neutron-induced fission cross sections (as well as the rates) increase with decreasing fission barriers and that $\sigma_{\mathrm{nf}}^{\mathrm{HM}}>\sigma_{\mathrm{nf}}^{\mathrm{TF}}>\sigma_{\mathrm{nf}}^{\mathrm{ETFSI}}$. The difference at low energies is due to the different mass predictions used (comparing calculations with TF fission barriers but different neutron separation energies $S_{\mathrm{n}}$ ). The small decrease in $S_{\mathrm{n}}$, when predictions for masses and fission barriers (based on the two sets of input data) are changed from $\mathrm{TF}+\mathrm{TF}$ to $\mathrm{FRDM}+\mathrm{TF}$, results in a decrease of the neutron-induced fission cross sections (accompanied by decreasing $(n, \gamma)$-cross sections). The same influence is illustrated by the cases where sets of consistent determinations for $S_{n}$ and $B_{f}$ are replaced by sets from different mass predictions. For example, different predictions of neutron separation energies were used for the same TF - fission barrier predictions. Therefore, cross sections for low energies differ significantly, but for higher $E$ the difference in cross section becomes much smaller for the same fission barriers (here we used TF barriers). The temperature averaged rates (Fig. 6, right panel) show the same dependence.

As expected, the rate sets calculated on the basis of FRDM masses and the TF model for masses and fission barriers are quite comparable (Fig. 7). On the other hand, when the sets include ETFSI vs. FRDM+TF or TF comparisons, the results can differ by up to eight orders of magnitude. The most extreme difference of ETFSI-based and other rates was obtained for nuclei with neutron numbers close to 184 , for which the ETFSI model 
I. V. Panov et al.: Neutron-induced rates...
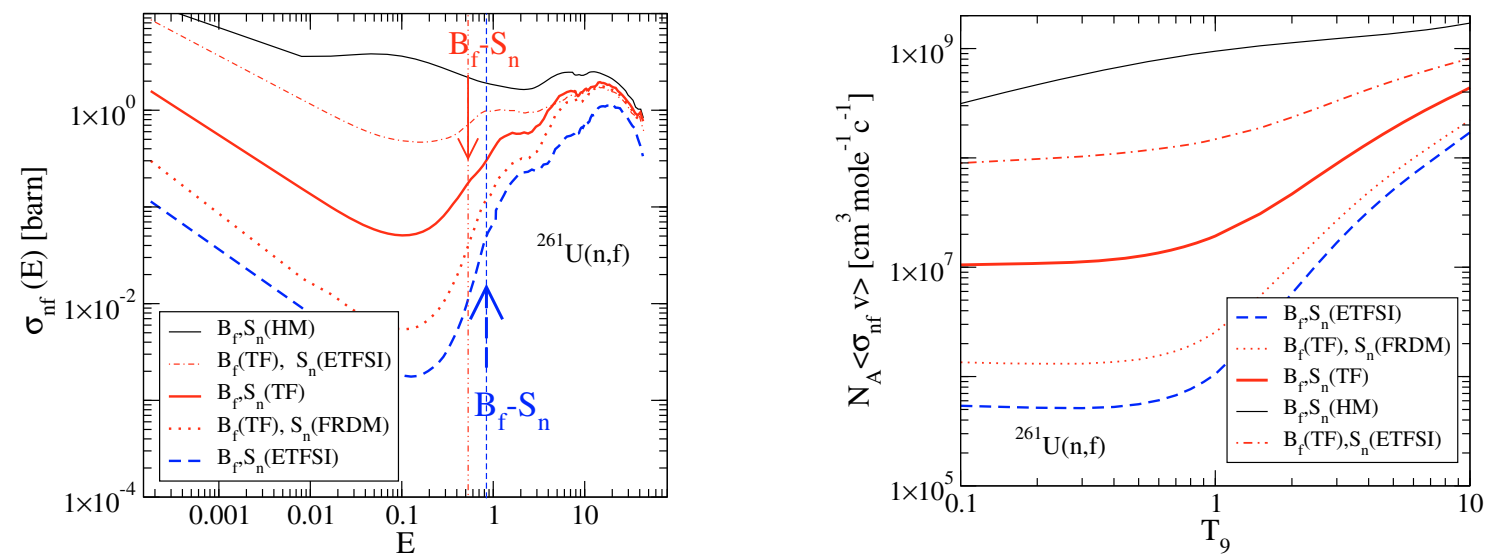

Fig. 6. Dependence of neutron-induced fission cross sections $\sigma_{n f}(E)$ (left) and rates $\lambda_{n f}=N_{A}\langle\sigma v\rangle$ (right) on mass- and fission barrier predictions for ${ }^{261} \mathrm{U}$. The sources for fission barriers $B_{f}$ and neutron separation energies $S_{n}$ (Howard and Möller, Thomas-Fermi, extended Thomas-Fermi, Finite Range Droplet Model) are indicated in the panel. Arrows show the difference between fission barrier and neutron separation energy $B_{f}-S_{n}$ for ETFSI (dashed line) and TF (full line) predictions.
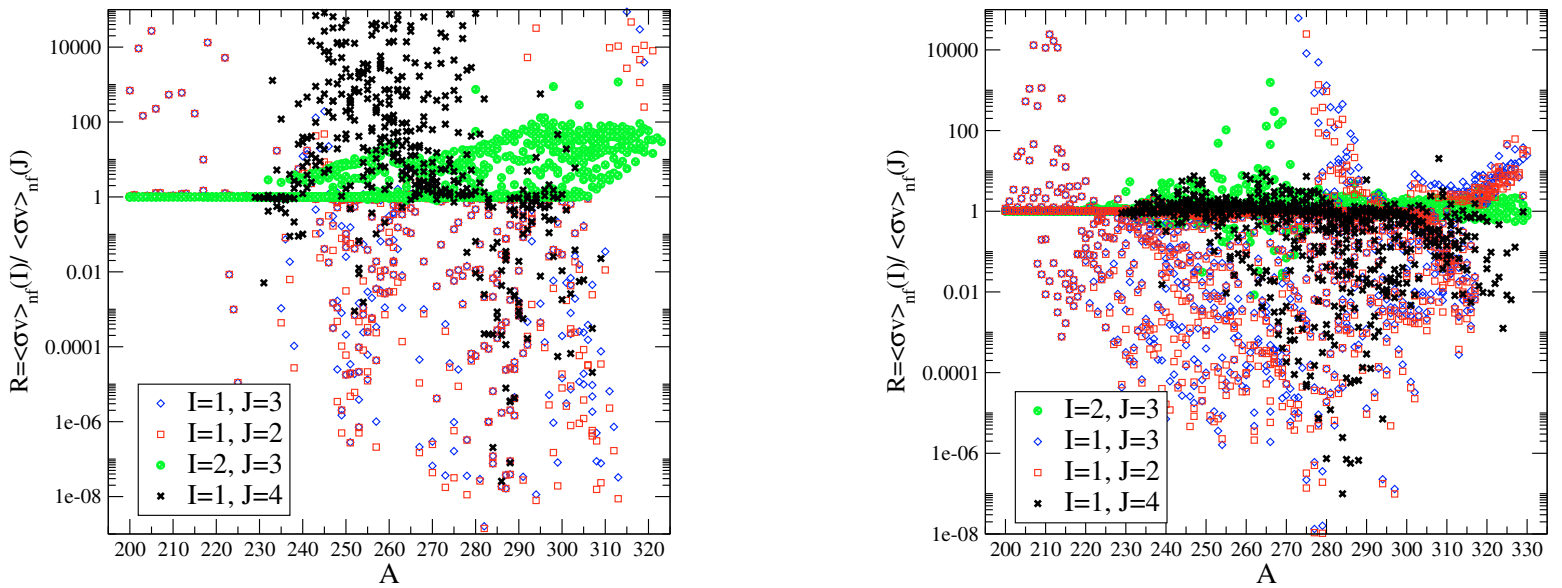

Fig. 7. Ratio $R=\frac{\langle\sigma v\rangle_{I}}{\langle\sigma v\rangle_{J}}$ of reaction rates for different fission barrier and mass predictions at temperatures $T_{9}=0.3(l e f t)$ and $T_{9}=10($ right $)$. The indexes $I, J$ define the mass/barrier predictions utilized in the calculations: ETFSI $(I, J=1)$, Thomas-Fermi $(I, J=2)$ or Thomas-Fermi for fission-barriers and FRDM for mass-predictions $(I, J=3$ ), Hartree-Fock-Bogolyubov (HFB-14) predictions $(I, J=4)$. As the Thomas-Fermi model and the Finite Range Droplet Model give similar mass predictions, the deviation for the combination $2 / 3$ is the smallest (and also decreases with temperature). However, the use of TF (or FRDM) vs. ETFSI (or HFB-14) predictions changes the rates drastically and thus the full range of possible magnitudes shown in Fig. 4 is obtained.

predicts very high fission barriers, leading to small fission rates. The difference between rates, calculated on the basis of ETFSI and HFB mass and fission barriers predictions is less than between ETFSI and TF, especially for higher $T_{9}$. For smaller $T_{9}$ the we can see that difference of rate values has the opposite sign for regions with $A \approx 240-280$ and $A>280$. This agrees with Fig. 5. Some of the combinations employed in Fig. 7 are shown to underline the huge problems which can arise when not using consistent data. The extrapolation of rate calculations to regions of very exotic nuclei is a hard task, and only further investigations can answer which kind of prediction is more preferable. At the moment the only choice is to test all available predictions in r-process calculations that are compared to astronomical abundance observations.

\section{Computed rate sets and mass ranges}

As we discussed in the introduction, our aim was to prepare sets of neutron-induced reaction rates, suited for calculations of the r-process up to very high atomic masses. In this sense, our rates extend the previously published ones for $Z<84$ (Rauscher \& Thielemann 2001) up to the region $84 \leq Z \leq Z_{\max }\left(Z_{\max }\right.$ depends on available nuclear input and varies from 102 for HFBpredictions to 118 for FRDM data). The exact range of nuclei for the different choices of mass and fission barrier input is given in Table 2.

Our calculations include all outgoing channels and give simultaneously predictions for neutron-induced fission, $(n, \gamma)$-, $(n, p)$-, and $(n, \alpha)$ rates. However, here we only provide tables and fits for the neutron-induced fission and neutron capture rates. Due to their small values for neutron-rich nuclei, the rates of the other calculated reaction types are not important in astrophysical applications.

The format of the tables is explained in Appendix A. The full electronic versions of the tables available on-line at the CDS include all rates for all mass predictions (Tables 3-6). The isotope and element ranges for which rates as well as rate fits are available are given in Table 2 for the FRDM, TF, ETFSI and 
A\&A 513, A61 (2010)

Table 2. Ranges of isotopes for which we calculated rates based on input from the models TF, FRDM, ETFSI and HFB. Given are the charge number $Z$ and the lower and upper limits $A_{\min }$ and $A_{\max }$ of the neutron number of the targets in the isotopic chain.

\begin{tabular}{c|cccccccc|c|cccccc}
\hline \hline \multicolumn{3}{c}{ TF-shc } & \multicolumn{2}{c}{ FRDM } & \multicolumn{3}{c}{ ETFSI } & \multicolumn{3}{c}{ HFB } & \multicolumn{4}{c}{ TF-shc } & \multicolumn{3}{c}{ FRDM } & \multicolumn{3}{c}{ ETFSI } \\
\hline$Z$ & $A_{\min }$ & $A_{\max }$ & $A_{\min }$ & $A_{\max }$ & $A_{\min }$ & $A_{\max }$ & $A_{\min }$ & $A_{\max }$ & $Z$ & $A_{\min }$ & $A_{\max }$ & $A_{\min }$ & $A_{\max }$ & $A_{\min }$ & $A_{\max }$ \\
\hline $84^{*}$ & 180 & 269 & 180 & 269 & 180 & 267 & 193 & 269 & 102 & 233 & 331 & 233 & 331 & 233 & 331 \\
$85^{*}$ & 185 & 270 & 185 & 270 & 185 & 270 & 193 & 270 & 103 & 240 & 335 & 240 & 334 & 240 & 328 \\
$86^{*}$ & 187 & 269 & 187 & 269 & 187 & 269 & 196 & 269 & 104 & 239 & 337 & 239 & 337 & 239 & 324 \\
$87^{*}$ & 190 & 280 & 190 & 280 & 190 & 280 & 200 & 278 & 105 & 242 & 337 & 242 & 331 & 242 & 330 \\
$88^{*}$ & 193 & 283 & 193 & 283 & 193 & 279 & 202 & 279 & 106 & 245 & 337 & 245 & 331 & 247 & 337 \\
$89^{*}$ & 196 & 288 & 196 & 288 & 196 & 284 & 206 & 285 & 107 & 248 & 337 & 248 & 332 & 243 & 337 \\
90 & 199 & 293 & 199 & 293 & 199 & 287 & 220 & 287 & 108 & 251 & 337 & 251 & 327 & 245 & 337 \\
91 & 212 & 296 & 212 & 296 & 212 & 288 & 229 & 289 & 109 & 254 & 337 & 254 & 327 & 247 & 337 \\
92 & 204 & 299 & 204 & 299 & 204 & 291 & 230 & 291 & 110 & 257 & 337 & 257 & 339 & 249 & 337 \\
93 & 220 & 302 & 220 & 302 & 220 & 293 & 232 & 293 & 111 & 260 & 337 & 260 & 331 & 251 & 329 \\
94 & 210 & 305 & 210 & 305 & 210 & 295 & 234 & 295 & 112 & 263 & 337 & 263 & 332 & 253 & 329 \\
95 & 215 & 309 & 215 & 308 & 215 & 297 & 238 & 297 & 113 & 267 & 337 & 267 & 327 & 255 & 329 \\
96 & 216 & 312 & 216 & 311 & 216 & 300 & 240 & 300 & 114 & 270 & 337 & 270 & 327 & 257 & 329 \\
97 & 225 & 315 & 225 & 314 & 225 & 303 & 243 & 315 & 115 & 273 & 337 & 273 & 337 & 275 & 329 \\
98 & 222 & 319 & 222 & 319 & 222 & 317 & 245 & 319 & 116 & 276 & 337 & 276 & 337 & - & - \\
99 & 225 & 322 & 225 & 322 & 225 & 320 & 250 & 322 & 117 & 279 & 337 & 279 & 337 & - & - \\
100 & 227 & 325 & 227 & 325 & 227 & 325 & 251 & 325 & 118 & 282 & 337 & 283 & 337 & - & - \\
101 & 239 & 328 & 239 & 328 & 239 & 326 & 254 & 328 & 119 & - & - & - & - & - & - \\
\hline
\end{tabular}

Notes. ${ }^{(*)}$ Given are the charge number $Z$ and the lower and upper limits $A_{\min }$ and $A_{\max }$ of the neutron number of the targets in the isotopic chain. For these chemical elements there are no HFB-predictions of fission barriers.

HFB mass predictions. This amounts to 2151 (ETFSI), 2637 (TF), 2400 (FRDM-masses, TF-barriers) and 1323 (HFB) involved nuclei. The partition functions for all isotopes are given on a grid of 24 temperatures: $T_{9}=0.1,0.15,0.2,0.3,0.4,0.5$, 0.6, 0.7, 0.8, 0.9, 1.0, 1.5, 2.0, 2.5, 3.0, 3.5, 4.0, 4.5, 5.0, 6.0, 7.0, $8.0,9.0,10.0$ and can be found in CDS's Tables 3-6 as well. We also provide the fit coefficient needed to compute the photodisintegration rate (see Sect. 4.1 ).

\subsection{The fits for neutron-induced and reverse rates}

Reaction rates have been calculated on a grid of 24 temperatures: $T_{9}=0.1,0.15,0.2,0.3,0.4,0.5,0.6,0.7,0.8,0.9,1.0,1.5,2.0$, $2.5,3.0,3.5,4.0,4.5,5.0,6.0,7.0,8.0,9.0,10.0$, same as for partition functions. These rates include the thermal modification in the stellar plasma, i.e. consider reactions from excited states in the target. For easy application in astrophysical investigations, these stellar rates were fitted with the same REACLIB parameterization as used for other reaction types earlier (Rauscher \& Thielemann 2000):

$$
\begin{array}{r}
N_{A}\langle\sigma v\rangle^{*}=\exp \left(a_{0}+a_{1} T_{9}^{-1}+a_{2} T_{9}^{-1 / 3}+a_{3} T_{9}^{1 / 3}+a_{4} T_{9}\right. \\
\left.+a_{5} T_{9}^{5 / 3}+a_{6} \ln T_{9}\right)
\end{array}
$$

with the seven open parameters $a_{0}-a_{6}$ and the stellar temperature $T_{9}$ given in $10^{9} \mathrm{~K}$. This parameterization proved to be flexible enough to accommodate the different temperature dependencies of the various reaction types across the fitted temperature range of $0.01 \leq T_{9} \leq 10$. Parameterizations of the present rates in the form used in Holmes et al. (1976) can be found in Appendices. The best fit was obtained by minimizing the deviation $\zeta$ (Eq. (5)) using the FUMILI code (Dymov et al. 2000).

The flexibility of the fitting function makes it prone to numerical problems outside the calculated range at low temperatures. In some cases they tend to diverge strongly. This difficulty can be avoided by additionally providing fit data at low temperatures to the calculated values by appropriately extrapolating the rates to lower temperatures. However, it has to be emphasized that the considered parameterization is only valid within the temperature range of $0.01 \leq T_{9} \leq 10.0$, although many fits will show a "proper" behavior down to lower temperature.

As a measure of the accuracy of a given fit, the quantity $\zeta$ (marked in tables and figures as Dev) is used. It is defined by

$\zeta=\frac{1}{24} \sum_{i=1}^{24}\left(\frac{r_{i}-f_{i}}{f_{i}}\right)^{2}$,

with $r$ being the original rate value as calculated at each of the 24 temperatures $T_{9}=0.1,0.15 \ldots 10.0$, and $f_{i}$ is the rate calculated from the fit at these temperatures. A small value of $\zeta$ indicates an accurate fit over the entire temperature range. Higher values of $\zeta$ are mainly caused by deviations at low temperatures, where rates are slow and a larger deviation is permissible. For the majority of nuclei the value of $\zeta$ is less than 1 and lies in the range $0.1-10^{-4}$ (see Fig. 9). We should mention here that the modified approximation formula by Cyburt \& Davids (2008) can probably give the better fit. Its accuracy can be high and should be applied for reactions, whose rates can be calculated with rather high accuracy. For our case fitting the average accuracy is not bad (see Fig. 8), and is much better than accuracy of calculations of neutron-induced rates for very neutron rich nuclei. We applied the approximation Eq. (4), used earlier in a number of previous predictions of neutron rates.

The temperature dependence of the rate can be one of two types, as illustrated in Fig. 8. These types of behavior can be understood when one recalls the discussion of Fig. 6. A fission transmission coefficient which is constant or slowly varying as a function of energy leads to an $(n, f)$-cross section which (similar to a pure neutron capture) shows a $1 / \sqrt{E}$ dependence, if s-wave dominated. Averaging such a cross section over a MaxwellBoltzmann distribution yields a constant rate. This situation occurs for example when the neutron bombarding energy leads to a compound nucleus energy above the lower and below the higher 

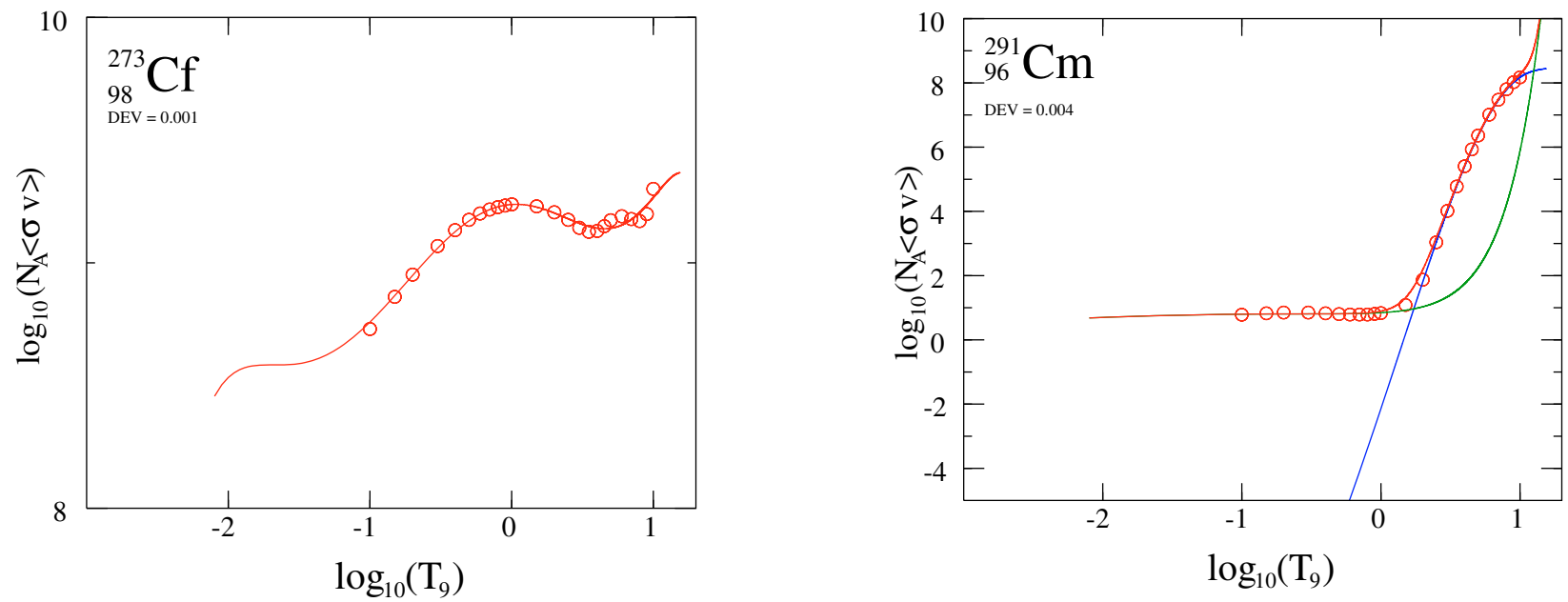

Fig. 8. Representative fits of neutron-induced fission rates $\left({ }^{273} \mathrm{Cf}\right.$ and $\left.{ }^{291} \mathrm{Cm}\right)$ for two types of temperature dependences. The general aim is to attain sufficient accuracy with one set of seven fitting coefficients (see Eq. (4)), as shown in the left curve. In some cases the superposition of two such sets is necessary (right panel) to correctly reproduce the low- as well as high-temperature behavior. A correct extrapolation to low temperatures $\left(0.01<T_{9}<0.1\right)$ is important to avoid unphysical abundance changes (see also the explanation of tables).
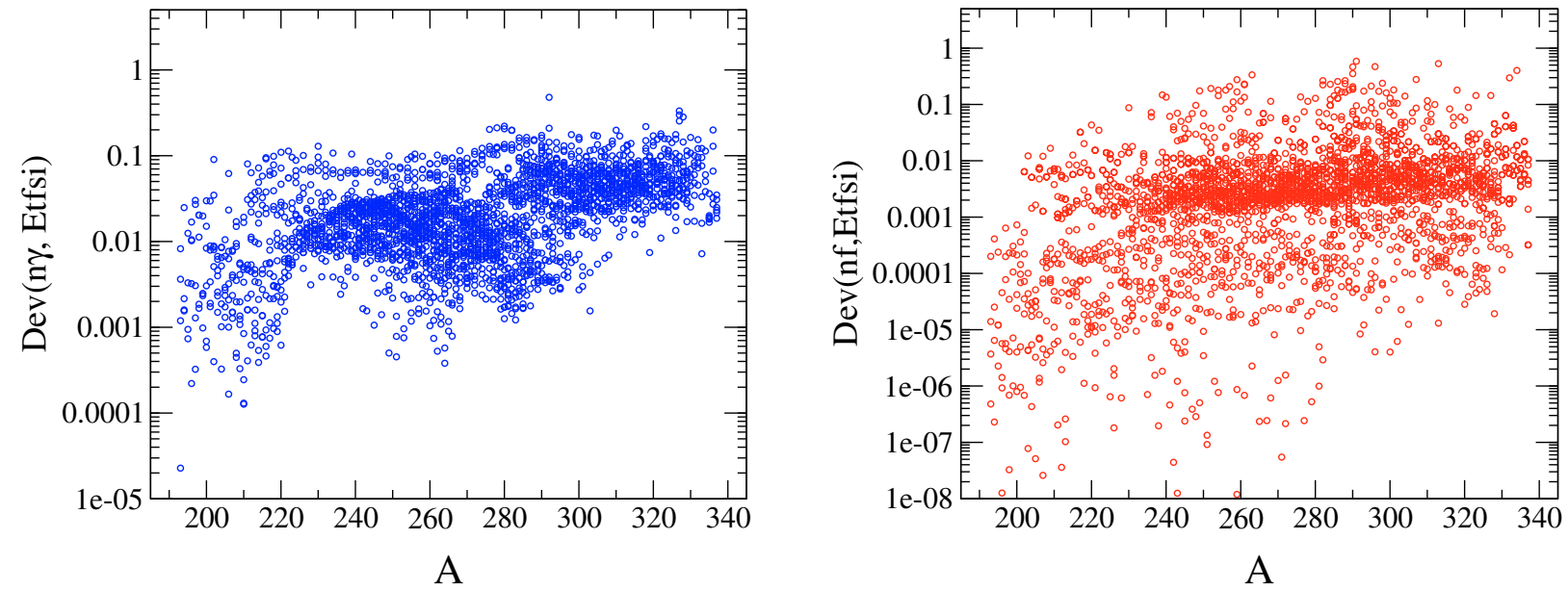

Fig. 9. The accuracy of fits is defined by a mean-square error (see Eq. (5)), shown here for $(n, \gamma)$-rate fits (left panel) and neutron-induced fission rate fits (right panel). We see that the upper boundary of errors is in the range of $10 \%$. This should be compared to the variations of these calculations (see Figs. 1 and 5).

barrier of a double-hump fission barrier. Then the lower barrier is open and the penetration through the remaining higher barrier is close to constant, but the height of the higher barrier determines the size of the $(n, f)$ cross section. This behavior is seen in Fig. 6 below about $0.1 \mathrm{MeV}$ (left panel). In the right panel the corresponding rate is shown and seen to be close to constant below about $T_{9}=1$.

In contrast, the opening of a fission barrier as a function of energy, i.e. an exponentially increasing transmission coefficient close to the barrier energy, leads to a sudden rise of the cross section, and consequently also of the rate as a function of temperature. We see this behavior for bombarding energies on the order of $B_{f}-S_{n}$ in the left panel of Fig. 6 and a similar behavior for the rates in the right panel. The size of this change from an almost constant to a steeply rising rate is a function of the barrier height. The right panel of Fig. 8 is a very representative example of such a case (almost constant rate at low temperatures and a steep rise by orders of magnitude at a critical temperature). On the other hand, when the compound energy is close to the barrier height already for small bombarding energies, the cross section and rate are already large at small energies (temperatures). Such an example can be seen in the left panel of Fig. 8, reflecting a double-hump behavior with small energy differences between the lower and higher barrier. For a discontinuity in the $T_{9}$-dependence (right panel), the fit was performed as a sum of two contributions and is given by two lines in the Table A.2 of the paper and CDS's Tables 7-18.

For all cases it is recommended to use the fits only down to the temperature $T_{9} \geq 0.01$. Moreover, close to the drip-line, the statistical model may not be applicable for reactions with low $Q$ value, even above that temperature. Although the fit may be good, the user should be aware of that possible complication.

\subsection{Photodisintegration rates and partition functions}

For a full implementation of the neutron captures in a reaction network, the inverse photodisintegration rates also have to 
be known. The photodisintegration rates (and their fits) are not explicitly given in the tables, but can be computed from the information contained therein. To calculate the reverse rate of the reaction $\mathrm{B}(\mathrm{n}, \gamma) \mathrm{D}$, i.e. the reaction $\mathrm{D}(\gamma, \mathrm{n}) \mathrm{B}$, the seven parameters $a_{0}^{\text {rev }}-a_{6}^{\text {rev }}$ are determined as follows:

$a_{0}^{\text {rev }}=a_{0}+\ln \left(9.8685 \times 10^{9}\left(\frac{A_{\mathrm{D}}-1}{A_{\mathrm{D}}}\right)^{1.5} \frac{2 J_{\mathrm{B}}+1}{2 J_{\mathrm{D}}+1}\left(2 J_{\mathrm{n}}+1\right)\right)$,

$a_{1}^{\mathrm{rev}}=a_{1}-11.6045 S_{n}$,

$a_{2}^{\text {rev }}=a_{2}$,

$a_{3}^{\mathrm{rev}}=a_{3}$,

$a_{4}^{\mathrm{rev}}=a_{4}$,

$a_{5}^{\mathrm{rev}}=a_{5}$,

$a_{6}^{\mathrm{rev}}=a_{6}+1.5$,

with $A_{\mathrm{D}}$ the mass number of nucleus $\mathrm{D}, J_{\mathrm{n}}$ the spin of neutron and $J_{\mathrm{B}}, J_{\mathrm{D}}$ the ground state spins of nuclei $\mathrm{B}$ and $\mathrm{D}$, respectively. These parameters for the reverse reaction are also given in the tables (see explanations in the appendices).

It is important to note that the value computed by applying Eq. (4) with the above coefficients has to be multiplied by the ratio of the partition functions for the residual and target nucleus $G_{\mathrm{B}} / G_{\mathrm{D}}$ to obtain the actual photodisintegration rate. Examples are shown in Appendix A. As it was shown in detail earlier (Rauscher \& Thielemann 2000), the temperature-dependent partition function $G\left(T^{*}\right)$ normalized to the ground state spin $J^{0}$ of a nucleus is defined as in Fowler et al. (1967)

$$
\begin{aligned}
\left(2 J^{0}+1\right) G\left(T^{*}\right)= & \sum_{\mu=0}^{\mu_{m}}\left(2 J^{\mu}+1\right) \mathrm{e}^{-E^{\mu} / k T^{*}} \\
& +\int_{E^{\mu_{m}}}^{E^{\max }} \sum_{J^{\mu}, \pi^{\mu}}\left(2 J^{\mu}+1\right) \mathrm{e}^{-\epsilon / k T^{*}} \rho\left(\epsilon, J^{\mu}, \pi^{\mu}\right) \mathrm{d} \epsilon,
\end{aligned}
$$

with $\rho$ being the level density and $\mu_{m}$ the last included experimentally known state. For the temperature range considered here, the maximum energy $E_{i}^{\max }$ above which there are no more significant contributions to the partition function is on the order of 20-30 MeV (Rauscher \& Thielemann 2000). The temperature dependent partition functions are available at the CDS, as well as the fit coefficients in Eq. (4) (Tables 7-18) for the $(n, \gamma)$, $(\gamma, n)$ and $(n, f)$ rates.

This subsection discussed photo-induced reaction rates as inverse reactions of neutron capture rates, which are presented in full detail in the present publication. Photo-induced reactions can also lead to fission when the Planck distribution of photons provides a significant fraction of photons with energies above the fission barrier. This reaction channel is not discussed here but its possible influence in r-process environments will be analyzed in a future investigation.

\section{Fission fragment distributions}

A proper inclusion of fission in r-process calculations also requires the knowledge of the resulting distribution of fission fragments, which have to be entered as reaction products. This by itself requires a major effort and will be presented with a thorough description of the treatment plus detailed fission yield distributions in a forthcoming paper. However, at the end of the present investigation we want to give a short outlook on how this topic will be approached.
In principle, such distributions are dependent on the excitation energy of the compound nucleus and thus would lead to a changing yield distribution for each bombarding energy. However, we found that the distributions vary smoothly and slowly as a function of the excitation energy. Thus, for the neutron energy range in astrophysical applications, the yield distribution at the neutron separation energy (i.e., for a vanishing neutron bombarding energy) is a very good approximation. This would permit us to multiply the fitted $(n, f)$-rates from the previous section with a static distribution of yields for all temperatures.

Here we only briefly show a few examples of such fission distributions. Properties of fission fragments, i.e., masses, atomic numbers, excitation and kinetic energies, were calculated based on the macro-microscopic approach (similar to the FRDM model) and the separability of compound-nucleus and fragment properties on the fission path (Schmidt et al. 2008; Wilkins et al. 1976). The original technical description of the fragmentformation model was published in Benlliure et al. (1998) and Kruglov et al. (2002). In the calculations shown here we used an updated description that will be the subject of a forthcoming publication.

In the model it is assumed that the different ways of splitting up the total mass are basically determined by the number of available transition states above the potential energy surface behind the outer saddle point. The macroscopic properties of the potential-energy landscape of the fissioning system are attributed to the strongly deformed fissioning system, which are deduced from mass distributions at high excitation energies (Rusanov 1997) and Langevin calculations (Nadtochy et al. 2005). The microscopic properties of the potential-energy landscape of the fissioning system are given by the qualitative features of the shell structure in the nascent fragments. They are determined from the observed features of the fission channels (Brosa et al. 1990) according to the procedure described by Schmidt et al. (2008). The dynamics of the fission process responsible for the fragment formation was considered in an approximate way: it was assumed that the phase space near the outer saddle point determines the mass asymmetry of the system, which is more or less frozen during the descent to scission. On the other hand, it was also assumed that the $N / Z$ collective degree of freedom is determined near the scission point. The excitation energies of the created fragments were calculated from the available excitation energy at the scission point and the deformation energies of the fragments at scission. The deformation energies of the fragments were assumed to be specific to the individual fission channels. They were deduced from experimental data (Wahl 1988; Böckstiegel et al. 2008, and references therein) on total kinetic energies and neutron yields. Kinetic energies were then calculated applying the energy conservation law. Finally, the two excited fission fragments are subject to particle (mostly neutrons) and $\gamma$ ray emission until they reach their ground state configurations. The de-excitation process was described in the framework of the statistical model as described in Kelić et al. (2008).

The left panel of Fig. 10 compares the mass distributions resulting from such an approach with the experimental data for ${ }^{238} \mathrm{U}$ (Nagy et al. 1978). We also show the yields computed using the empirical parameterizations developed by Panov et al. (2008) and Kodama \& Takahashi (1975), which previously were used in r-process calculations. In order to give an impression of the impact of the results for r-process nuclei, the two right panels of Fig. 10 show the mass distributions resulting from the fission of ${ }^{282} \mathrm{Cm}$ and ${ }^{292} \mathrm{Cf}$. For these nuclei, clear differences appear between the phenomenological 

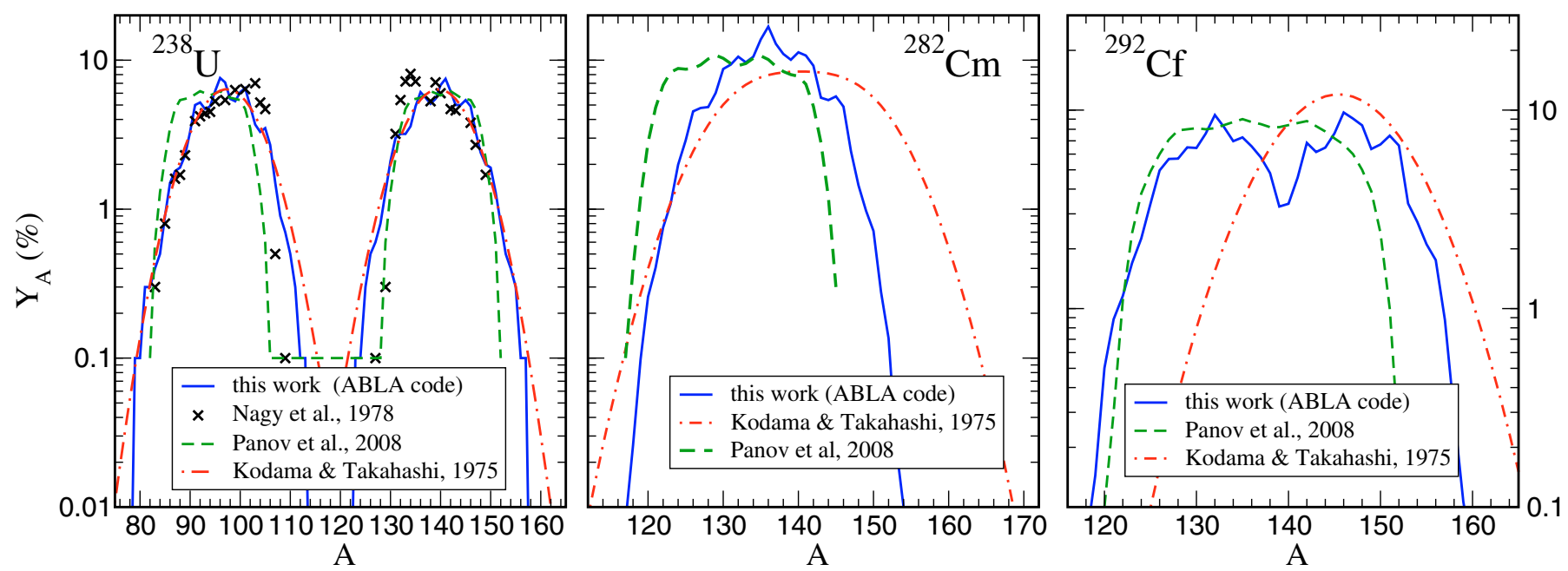

Fig. 10. The final mass distributions of fission fragments for the compound nuclei (after neutron capture) ${ }^{238} \mathrm{U},{ }^{282} \mathrm{Cm}$ and ${ }^{292} \mathrm{Cf}$. The distributions were computed with the ABLA code (Kelić et al. 2008) as described in the text. In addition, we show the yields computed with the phenomenological parameterizations of Panov et al. (2008) and Kodama \& Takahashi (1975), as well as experimental data (crosses).

approaches (Panov et al. 2008; Kodama \& Takahashi 1975) and the macroscopic-microscopic approach described above. For application in r-process simulations the $(n, f)$-reaction rates (which are the focus of the present paper) will have to be multiplied by the displayed percentages for obtaining a production rate of a specific fragment nucleus (Kelić et al. 2005; MartínezPinedo et al. 2007; Panov et al. 2008).

\section{Summary and conclusions}

We provide predictions of neutron-induced fission rates and $(n, \gamma)$-rates for a wide range of astrophysical temperatures $\left({ }^{8} \leq\right.$ $T(\mathrm{~K}) \leq 10^{10}$ ) and targets (proton- to neutron- drip-line for $84 \leq Z \leq 118$, i.e. from Po to Uuo ) in the framework of the Wolfenstein-Hauser-Feshbach model, making use of a variety of different mass and fission barrier predictions (Myers \& Swiatecki 1999; Mamdouh et al. 2001; Aboussir et al. 1995; Möller et al. 1995; Howard \& Möller 1980; Goriely et al. 2009). The astrophysical (stellar) reaction rates were fitted as in previous works (Thielemann et al. 1987; Rauscher \& Thielemann 2000) in the common REACLIB seven parameter form, and these parameters are also tabulated. This provides the basis for r-process nucleosynthesis calculations where the abundance predictions for the highest mass numbers as well as the effect of fission cycling are strongly dependent on the interplay of neutron capture and fission.

In order to give an impression of the reliability of the results, we compared them with experiment and with available independent predictions before exploring the currently unreachable regions of the nuclear chart with a variety of theoretical predictions for nuclear masses and fission barriers (FRDM, ETFSI, TF, HFB). An extended comparison of neutron-induced fission rates with experiment and with available independent predictions was done. The dependence of rates on nuclear input data, most of all fission barriers, is high. Astrophysical nucleosynthesis yield predictions, especially in the transuranium region, should take into account these large differences in order to explore the variations involved. For this reason extended tables for neutron-induced fission rates as well neutron capture rates are presented for different mass and fission barrier predictions in fitted form for nucleosynthesis calculations. Their structure is given in the Appendix A (note that the full rate and fit tables are available at the CDS). Given that fission predictions far from stability have not been tested yet, and even close to stability none of the existing models has yet been proven to be superior (see Fig. 3). Nucleosynthesis calculations should probably continue to use a variety of these models. A further requirement for nucleosynthesis modeling in the region of fissioning nuclei is the knowledge of the mass distribution of fission products. This work is in progress (see Sect. 5).

Acknowledgements. The authors thank E. Kolbe, K.-L. Kratz, K. Langanke, P. Möller, D. K. Nadyozhin, and B. Pfeiffer for useful discussions. This work was supported by the Swiss National Science Foundation (SCOPES projects No. IB7320-110996 and No. IZ73Z0-128180/1) and grant 2000-105328. I.P. and I.K. were partly supported by Federal Programm "Scientific and pedagogical specialists of innovation Russia", contract number 02.740.11.0250 and RFBRgrant 09-02-12168-ofi_m.

\section{Appendix A: Explanation of the tables and examples of how to use them}

This section is intended to help with interpreting the information given in the electronic tables. We also give examples for calculating the reaction rate for a given reaction and its inverse reaction.

Table A.1 shows which information is contained in the rate Tables 3-6 for different mass predictions, available at the CDS (Table A.1 illustrated some lines from CDS Table 3: rates on the basis of ETFSI-predictions). The organization of Tables 3-6 with calculated rates for 24 different values of $T_{9}$ is extremely simple - all data are in eight columns. The columns give, in this order, the target element, the atomic mass number $A$ of the target, the temperature in $T_{9}$, the partition function of the target, the neutron-induced fission rate for the ground state, the neutroninduced fission rate with thermally populated target states, the neutron capture rate for the ground state, and the neutron capture rate with thermally populated target states (units of all rates: $\left.\mathrm{cm}^{-3} \mathrm{~mole}^{-1} \mathrm{c}^{-1}\right)$.

The rates in Tables 3-6 were calculated on the basis of different mass and fission barrier predictions: ETFSI, TF, 
Table A.1. Example of format of rates presented in the Tables 3-6, available at the CDS: reaction rates $N_{A}\langle\sigma v\rangle$ and partition functions show a subset of calculation based on ETFSI predictions.

\begin{tabular}{cccccccc}
\hline \hline \multicolumn{2}{l}{ mother(A,Z) } & $T_{9}$ & p.f. & $(n, f)$ & $(n, f)^{*}$ & $(n, g)$ & $(n, g)^{*}$ \\
\hline Cf & 273 & 0.10 & $1.00 \mathrm{D}+00$ & $5.07 \mathrm{D}+08$ & $5.90 \mathrm{D}+08$ & $2.89 \mathrm{D}+04$ & $3.32 \mathrm{D}+04$ \\
$\mathrm{Cf}$ & 273 & 0.15 & $1.01 \mathrm{D}+00$ & $6.59 \mathrm{D}+08$ & $8.13 \mathrm{D}+08$ & $3.71 \mathrm{D}+04$ & $4.50 \mathrm{D}+04$ \\
$\mathrm{Cf}$ & 273 & 0.20 & $1.02 \mathrm{D}+00$ & $7.81 \mathrm{D}+08$ & $1.01 \mathrm{D}+09$ & $4.35 \mathrm{D}+04$ & $5.50 \mathrm{D}+04$ \\
$\mathrm{Cf}$ & 273 & 0.30 & $1.08 \mathrm{D}+00$ & $9.70 \mathrm{D}+08$ & $1.33 \mathrm{D}+09$ & $5.28 \mathrm{D}+04$ & $6.99 \mathrm{D}+04$ \\
$\ldots$ & $\cdots$ & $\ldots$ & $\ldots$ & $\ldots$ & $\ldots$ & $\ldots$ & $\ldots$ \\
$\mathrm{Cf}$ & 273 & 7.00 & $7.86 \mathrm{D}+04$ & $1.89 \mathrm{D}+09$ & $1.57 \mathrm{D}+09$ & $5.70 \mathrm{D}+04$ & $9.86 \mathrm{D}+01$ \\
$\mathrm{Cf}$ & 273 & 8.00 & $9.13 \mathrm{D}+05$ & $1.93 \mathrm{D}+09$ & $1.53 \mathrm{D}+09$ & $5.41 \mathrm{D}+04$ & $1.47 \mathrm{D}+01$ \\
$\mathrm{Cf}$ & 273 & 9.00 & $1.04 \mathrm{D}+07$ & $1.98 \mathrm{D}+09$ & $1.60 \mathrm{D}+09$ & $5.14 \mathrm{D}+04$ & $2.20 \mathrm{D}+00$ \\
$\mathrm{Cf}$ & 273 & 10.00 & $1.03 \mathrm{D}+08$ & $2.03 \mathrm{D}+09$ & $1.97 \mathrm{D}+09$ & $4.89 \mathrm{D}+04$ & $3.69 \mathrm{D}-01$ \\
$\cdots$ & $\cdots$ & $\cdots$ & $\cdots$ & $\ldots$ & $\ldots$ & $\ldots$ & $\cdots$ \\
$\mathrm{Cf}$ & 274 & 7.00 & $3.87 \mathrm{E}+05$ & $1.75 \mathrm{E}+08$ & $1.68 \mathrm{E}+09$ & $4.04 \mathrm{E}+06$ & $5.85 \mathrm{E}+02$ \\
\hline
\end{tabular}

Table A.2. Parameterization of the $(n, \gamma)-,(\gamma, n)$ - and $(n, f)$-rates, available at the CDS Tables 6-18.

Example of Table $7-(n, \gamma)$-rate fits on the basis of ETFSI mass-model predictions.

\begin{tabular}{|c|c|c|c|c|c|c|c|c|c|}
\hline mother(A,Z) & $i_{\text {fit }}$ & $a_{0}$ & $a_{1}$ & $a_{2}$ & $a_{3}$ & $a_{4}$ & $a_{5}$ & $a_{6}$ & Dev \\
\hline cf 26898 & 0 & $-1.45 \mathrm{E}+01$ & $0.00 \mathrm{E}+00$ & $-2.94 \mathrm{E}+00$ & $3.00 \mathrm{E}+01$ & $-4.69 \mathrm{E}+00$ & $1.40 \mathrm{E}-01$ & $-6.04 \mathrm{E}+00$ & $3.9 \mathrm{E}-02$ \\
\hline cf 26998 & 0 & $2.05 \mathrm{E}+01$ & $1.43 \mathrm{E}-02$ & $-6.95 \mathrm{E}-01$ & $-3.73 E+00$ & $1.60 \mathrm{E}-01$ & $-3.13 \mathrm{E}-01$ & 7.64E-01 & $5.0 \mathrm{E}-02$ \\
\hline cf 27098 & 0 & $-2.03 E+01$ & $0.00 \mathrm{E}+00$ & $-3.86 \mathrm{E}+00$ & $3.56 \mathrm{E}+01$ & $-5.02 \mathrm{E}+00$ & $1.90 \mathrm{E}-01$ & $-7.77 \mathrm{E}+00$ & $1.9 \mathrm{E}-02$ \\
\hline cf 27198 & 0 & $-6.15 \mathrm{E}+00$ & $1.68 \mathrm{E}-02$ & $-3.20 \mathrm{E}+00$ & $1.94 \mathrm{E}+01$ & $-2.75 \mathrm{E}+00$ & $-3.24 \mathrm{E}-03$ & $-4.49 \mathrm{E}+00$ & $1.1 \mathrm{E}-02$ \\
\hline cf 27298 & 0 & $-1.69 \mathrm{E}+01$ & $0.00 \mathrm{E}+00$ & $-4.68 \mathrm{E}+00$ & $4.14 \mathrm{E}+01$ & $-5.82 \mathrm{E}+00$ & $1.64 \mathrm{E}-01$ & $-9.39 E+00$ & $3.6 \mathrm{E}-02$ \\
\hline cf 27398 & 0 & $2.62 \mathrm{E}+00$ & $1.71 \mathrm{E}-02$ & $-2.67 E+00$ & $1.32 \mathrm{E}+01$ & $-1.60 \mathrm{E}+00$ & $-1.64 \mathrm{E}-01$ & $-3.27 \mathrm{E}+00$ & 2.2E-02 \\
\hline cf 27498 & 0 & $-1.87 \mathrm{E}+01$ & $0.00 \mathrm{E}+00$ & $-5.35 \mathrm{E}+00$ & $4.34 \mathrm{E}+01$ & $-4.94 \mathrm{E}+00$ & $2.04 \mathrm{E}-02$ & $-1.03 \mathrm{E}+01$ & $4.9 \mathrm{E}-02$ \\
\hline cf 27598 & 0 & $2.43 \mathrm{E}+00$ & $0.00 \mathrm{E}+00$ & $-1.74 \mathrm{E}+00$ & $1.61 \mathrm{E}+01$ & $-2.19 \mathrm{E}+00$ & $-1.24 \mathrm{E}-01$ & $-3.45 E+00$ & $1.9 \mathrm{E}-02$ \\
\hline cf 27698 & 0 & $-1.50 \mathrm{E}+01$ & $0.00 \mathrm{E}+00$ & $-4.72 E+00$ & $3.79 \mathrm{E}+01$ & $-4.15 E+00$ & $-3.04 \mathrm{E}-02$ & $-9.12 \mathrm{E}+00$ & $3.8 \mathrm{E}-02$ \\
\hline cf 27798 & 0 & $-5.42 \mathrm{E}+00$ & $0.00 \mathrm{E}+00$ & $-3.49 \mathrm{E}+00$ & $2.64 \mathrm{E}+01$ & $-2.77 \mathrm{E}+00$ & $-1.12 \mathrm{E}-01$ & $-6.64 \mathrm{E}+00$ & $3.1 \mathrm{E}-02$ \\
\hline
\end{tabular}

Example of Table 11 - reverse $(\gamma, n)$-rate fits on the basis of ETFSI mass-model predictions.

\begin{tabular}{|c|c|c|c|c|c|c|c|c|}
\hline mother(A,Z) & $i_{\text {fit }}$ & $a_{0}^{\mathrm{rev}}$ & $a_{1}^{\mathrm{rev}}$ & $a_{2}^{\mathrm{rev}}$ & $a_{3}^{\mathrm{rev}}$ & $a_{4}^{\mathrm{rev}}$ & $a_{5}^{\mathrm{rev}}$ & $a_{6}^{\mathrm{rev}}$ \\
\hline cf 26998 & 0 & $1.05 \mathrm{E}+01$ & $-3.46 \mathrm{E}+01$ & $-2.94 \mathrm{E}+00$ & $3.00 \mathrm{E}+01$ & $-4.69 \mathrm{E}+00$ & $1.40 \mathrm{E}-01$ & $-4.54 \mathrm{E}+00$ \\
\hline cf 27098 & 0 & $4.28 \mathrm{E}+01$ & $-5.82 \mathrm{E}+01$ & $-6.95 \mathrm{E}-01$ & $-3.73 E+00$ & 1.60E-01 & $-3.13 \mathrm{E}-01$ & $2.26 \mathrm{E}+00$ \\
\hline cf 27198 & 0 & $4.72 \mathrm{E}+00$ & $-4.43 E+01$ & $-3.86 \mathrm{E}+00$ & $3.56 \mathrm{E}+01$ & $-5.02 \mathrm{E}+00$ & $1.90 \mathrm{E}-01$ & $-6.27 E+00$ \\
\hline cf 27298 & 0 & $1.61 \mathrm{E}+01$ & $-5.62 \mathrm{E}+01$ & $-3.20 \mathrm{E}+00$ & $1.94 \mathrm{E}+01$ & $-2.75 E+00$ & $-3.24 \mathrm{E}-03$ & $-2.99 \mathrm{E}+00$ \\
\hline cf 27398 & 0 & $8.14 \mathrm{E}+00$ & $-3.73 E+01$ & $-4.68 \mathrm{E}+00$ & $4.14 \mathrm{E}+01$ & $-5.82 \mathrm{E}+00$ & 1.64E-01 & $-7.89 \mathrm{E}+00$ \\
\hline cf 27498 & 0 & $2.49 \mathrm{E}+01$ & $-5.28 \mathrm{E}+01$ & $-2.67 \mathrm{E}+00$ & $1.32 \mathrm{E}+01$ & $-1.60 \mathrm{E}+00$ & $-1.64 \mathrm{E}-01$ & $-1.77 \mathrm{E}+00$ \\
\hline 27598 & 0 & $6.30 \mathrm{E}+00$ & $-3.57 \mathrm{E}+01$ & $-5.35 \mathrm{E}+00$ & $4.34 \mathrm{E}+01$ & $-4.94 \mathrm{E}+00$ & $2.04 \mathrm{E}-02$ & $-8.87 \mathrm{E}+00$ \\
\hline cf 27698 & 0 & $2.47 \mathrm{E}+01$ & $-5.01 \mathrm{E}+01$ & $-1.74 \mathrm{E}+00$ & $1.61 \mathrm{E}+01$ & $-2.19 \mathrm{E}+00$ & $-1.24 \mathrm{E}-01$ & $-1.95 \mathrm{E}+00$ \\
\hline cf 27798 & 0 & $1.00 \mathrm{E}+01$ & $-3.66 \mathrm{E}+01$ & $-4.72 E+00$ & $3.79 E+01$ & $-4.15 E+00$ & $-3.04 \mathrm{E}-02$ & $-7.62 E+00$ \\
\hline cf 27898 & 0 & $1.68 \mathrm{E}+01$ & $-5.02 \mathrm{E}+01$ & $-3.49 \mathrm{E}+00$ & $2.64 \mathrm{E}+01$ & $-2.77 \mathrm{E}+00$ & $-1.12 \mathrm{E}-01$ & $-5.14 \mathrm{E}+00$ \\
\hline
\end{tabular}

FRDM(masses)+TF(barriers) and HFB respectively (see main text of the paper for details).

The entries are denoted as follows:

mother mother nucleus (target),

$T_{9} \quad$ stellar temperature in $10^{9} \mathrm{~K}$,

p.f. nuclear partition function,

$(n, g) \quad(n, \gamma)$-rate, $N_{A}\left\langle\sigma_{n \gamma} v\right\rangle$

$(n, g)^{*} \quad(n, \gamma)$-rate, $N_{A}\left\langle\sigma_{n \gamma} v\right\rangle$, with thermally populated target levels,

$(n, f) \quad\left(n\right.$, fission)-rate, $N_{A}\left\langle\sigma_{n f} v\right\rangle$

$(n, f)^{*} \quad\left(n\right.$, fission)-rate, $N_{A}\left\langle\sigma_{n f} v\right\rangle$, with thermally populated target levels.

Table A.2 shows which information is contained in the fit Tables 7-18, available at the CDS. The following information is provided:

mother reaction target
Dev fit accuracy $\zeta$ (Eq. (5))

$a_{0} \ldots a_{6} \quad$ seven fit parameters for the forward rate $a_{0}^{\text {rev }} \ldots a_{6}^{\text {rev }}$ seven fit parameters for the reverse rate fit (see Sect. 4.2).

The fitting coefficients for the (neutron, $\gamma$ )-, $(\gamma, n)-$, and neutron-induced fission rates with different mass and fissionbarrier predictions are placed in the Tables 7-18. The columns in Tables 7-18 are organized as follows: target element, atomic mass number $A$ of the target, target charge number $Z$, the number of fitting curves $i_{\text {fit }}$, seven coefficients of the forward reaction $a_{i}$, and the mean square error.

A value $i_{\text {fit }}=0$ means that there is only one seven-parameter set to fit the rate. Values $i_{\text {fit }}>0$ give the number of parameter sets which have to be added up to yield the final rate, i.e. the rate $r$ is calculated as $r=\sum_{i} r_{i}$, with each $r_{i}$ computed from the $i$ th parameter set and using Eq. (4). 
Table A.2. continued.

Example of Table 15 - neutron-induced fission rate fits on the basis of ETFSI mass-model predictions.

\begin{tabular}{|c|c|c|c|c|c|c|c|c|c|}
\hline mother(A,Z) & $i_{\text {fit }}$ & $a_{0}$ & $a_{1}$ & $a_{2}$ & $a_{3}$ & $a_{4}$ & $a_{5}$ & $a_{6}$ & Dev \\
\hline cf 26898 & 0 & $3.13 \mathrm{E}+01$ & $-6.04 \mathrm{E}-02$ & $8.24 \mathrm{E}+00$ & $-1.81 \mathrm{E}+01$ & $2.22 \mathrm{E}-01$ & $7.09 \mathrm{E}-02$ & $8.65 \mathrm{E}+00$ & $\overline{5.2 \mathrm{E}-03}$ \\
\hline cf 26998 & 0 & $3.38 \mathrm{E}+01$ & $0.00 \mathrm{E}+00$ & $2.22 \mathrm{E}+00$ & $-1.59 \mathrm{E}+01$ & $1.39 \mathrm{E}+00$ & $-7.64 \mathrm{E}-02$ & $4.52 \mathrm{E}+00$ & $4.5 \mathrm{E}-03$ \\
\hline cf 27098 & 0 & $9.75 E+00$ & $3.15 \mathrm{E}-03$ & $-1.49 E+00$ & $1.34 \mathrm{E}+01$ & $-1.77 E+00$ & $1.59 \mathrm{E}-01$ & $-2.78 \mathrm{E}+00$ & 7.7E-03 \\
\hline cf 27198 & 0 & $3.84 \mathrm{E}+01$ & $-5.73 \mathrm{E}-02$ & $8.81 \mathrm{E}+00$ & $-2.75 E+01$ & $1.86 \mathrm{E}+00$ & $-9.37 \mathrm{E}-02$ & $1.04 \mathrm{E}+01$ & $1.5 \mathrm{E}-03$ \\
\hline cf 27298 & 0 & $-1.30 \mathrm{E}+01$ & $0.00 \mathrm{E}+00$ & $-4.24 \mathrm{E}+00$ & $4.22 \mathrm{E}+01$ & $-5.66 \mathrm{E}+00$ & 4.90E-01 & $-9.00 \mathrm{E}+00$ & $1.7 \mathrm{E}-02$ \\
\hline cf 27398 & 0 & $3.87 \mathrm{E}+01$ & $-5.66 \mathrm{E}-02$ & $8.78 \mathrm{E}+00$ & $-2.78 \mathrm{E}+01$ & $1.89 \mathrm{E}+00$ & $-9.66 \mathrm{E}-02$ & $1.04 \mathrm{E}+01$ & $1.2 \mathrm{E}-03$ \\
\hline cf 27498 & 1 & $-4.50 \mathrm{E}+01$ & $0.00 \mathrm{E}+00$ & $0.00 \mathrm{E}+00$ & $6.69 \mathrm{E}+01$ & $-8.82 \mathrm{E}+00$ & $6.52 \mathrm{E}-01$ & $-8.66 \mathrm{E}+00$ & $1.5 \mathrm{E}-03$ \\
\hline cf 27498 & 0 & $3.45 \mathrm{E}+01$ & $0.00 \mathrm{E}+00$ & $0.00 \mathrm{E}+00$ & $-3.97 \mathrm{E}+01$ & $3.13 \mathrm{E}+01$ & $-1.60 \mathrm{E}+01$ & $3.53 \mathrm{E}+00$ & $1.5 \mathrm{E}-03$ \\
\hline cf 27598 & 0 & $8.34 \mathrm{E}+00$ & $1.13 \mathrm{E}-02$ & $-2.48 \mathrm{E}+00$ & $1.50 \mathrm{E}+01$ & $-1.35 E+00$ & 8.30E-02 & $-3.67 \mathrm{E}+00$ & $1.1 \mathrm{E}-03$ \\
\hline cf 27698 & 1 & $-3.74 \mathrm{E}+01$ & $0.00 \mathrm{E}+00$ & $0.00 \mathrm{E}+00$ & $5.39 \mathrm{E}+01$ & $-8.74 \mathrm{E}+00$ & $6.38 \mathrm{E}-01$ & $0.00 \mathrm{E}+00$ & $1.5 \mathrm{E}-02$ \\
\hline cf 27698 & 0 & $8.18 \mathrm{E}+00$ & $0.00 \mathrm{E}+00$ & $0.00 \mathrm{E}+00$ & $-2.88 \mathrm{E}+00$ & $6.77 \mathrm{E}+00$ & $-2.68 \mathrm{E}+00$ & $0.00 \mathrm{E}+00$ & $1.5 \mathrm{E}-02$ \\
\hline cf 27798 & 1 & $8.74 \mathrm{E}+01$ & $0.00 \mathrm{E}+00$ & $0.00 \mathrm{E}+00$ & $-7.63 \mathrm{E}+01$ & $3.03 \mathrm{E}+00$ & $-7.28 \mathrm{E}-02$ & $3.10 \mathrm{E}+01$ & $1.2 \mathrm{E}-03$ \\
\hline cf 27798 & 0 & $1.87 \mathrm{E}+01$ & $0.00 \mathrm{E}+00$ & $0.00 \mathrm{E}+00$ & $-4.86 \mathrm{E}+00$ & $4.23 \mathrm{E}+00$ & $-1.66 \mathrm{E}+00$ & $4.15 \mathrm{E}-01$ & $1.2 \mathrm{E}-03$ \\
\hline
\end{tabular}

The examples of Tables 3-6 are given for 1 isotope for 24 values of $T_{9}$, and for Tables $7-18$ - for 10 isotopes. Note that the tables of CDS show the values with an accuracy of seven digits.

Below we give two examples for calculating a rate at $T_{9}=$ 7.0 with the fit parameters listed in the tables.

The first example is the reaction ${ }^{273} \mathrm{Cf}(n, f)$. In Table A. 2 one finds the parameters $a_{0}=38.72, a_{1}=-0.057, a_{2}=8.78, a_{3}=$ $-27.85, a_{4}=1.90, a_{5}=-0.097, a_{6}=10.45$. With the help of Eq. (4) one calculates $N_{A}\langle\sigma v\rangle_{\text {fit }}^{*}=1.574 \times 10^{9} \mathrm{~cm}^{3} \mathrm{~s}^{-1} \mathrm{~mole}^{-1}$ or $\log _{10}\left(N_{A}\langle\sigma v\rangle_{\text {fit }}^{*}\right)=9.20$ at $T_{9}=7.0$.

The second example is for the rates of the capture reaction ${ }^{273} \mathrm{Cf}(\mathrm{n}, \gamma){ }^{274} \mathrm{Cf}$ and its reverse reaction. Similar to the above example, using the parameters from Table A.2 and Eq. (4) the capture rate is easily found to be $N_{A}\langle\sigma v\rangle_{\text {fit }}^{*}=120 \mathrm{~cm}^{3} \mathrm{~s}^{-1} \mathrm{~mole}^{-1}$. With the reverse parameters the first value in the determination of the reverse rate is found to be $\lambda_{\gamma}{ }^{\prime}=5.0 \times 10^{9} \mathrm{~s}^{-1}$ at $T_{9}=7.0$. In order to obtain the actual value of the reverse rate, one first has to determine the ratio of the partition functions $G^{273} \mathrm{Cf} / G^{274} \mathrm{Cf}=4.58 \times 10^{5} / 3.87 \times 10^{5}=1.18($ see Table A. 1 and Sect. 4.2). The value $\lambda^{\prime}$ has to be multiplied by this ratio to derive the photodisintegration rate $\lambda$ :

$\lambda_{\gamma}=\lambda_{\gamma}^{\prime} \frac{G_{273} \mathrm{Cf}}{G_{274} \mathrm{Cf}}=\lambda_{\gamma}^{\prime} \times 1.18=5.9 \times 10^{9} \quad \mathrm{~s}^{-1}$.

The values of the partition functions at $T_{9}$ are also given in the online Tables 3-6. Note that the procedure is always the same as described above, regardless of whether it is an exoergic or an endoergic reaction.

\section{References}

Aboussir, Y., Pearson, J. M., Dutta, A. K., \& Tondeur, F. 1995, At. Data Nucl. Data Tables, 61, 127

Aikawa, M., Arnould, M., Goriely, S., Jorissen, A., \& Takahashi, K. 2005, A\&A, 441, 1195

\section{Arnould, M. 1972, A\&A, 19, 92}

Belgya, T., Bersillon, O., \& Capote, R. 2006, in Handbook for calculations of nuclear reaction data, RIPL-2, IAEA-TECDOC-1506 (Vienna: IAEA Vienna), Available online at http: //www-nds.iaea.org/RIPL-2/

Benlliure, J., Grewe, A., de Jong, M., Schmidt, K.-H., \& Zhdanov, S. 1998, Nucl. Phys. A, 628, 458

Bjornholm, S., \& Lynn, J. E. 1980, Rev. of Mod. Phys., 52, 725

Böckstiegel, C., Steinhäuser, S., Schmidt, K.-H., et al. 2008, Nucl. Phys., A802, 12

Brosa, U., Grossmann, S., \& Müller, A. 1990, Phys. Rep., 197, 167

Cowan, J., Pfeiffer, B., Kratz, K.-L., et al. 1999, ApJ, 521, 194
Cowan, J. J., Thielemann, F.-K., \& Truran, J. W. 1991, Phys. Rep., 208, 267 Cyburt, R. H., \& Davids, B. 2008, Phys. Rev. C., 78, 064614

Dymov, S. N., Kurbatov, V. S., Silin, I. N., \& Yaschenko, S. V. 2000, Nucl. Instr. Meth. Phys. Res. A, 440, 43

Experimental nuclear reaction data library. 2009, EXFOR:

http://www-nds.iaea.or.at/exfor, IAEA, NDC

Firestone, R. B., \& Shirley, V. S. 1996, Table of Isotopes, 8th ed. (New York: Wiley)

Fowler, W. A. 1974, QJRAS, 15, 82

Fowler, W. A., Caughlan, G. E., \& Zimmerman, B. A. 1967, ARA\&A, 5, 525

Freiburghaus, C., Rembges, J.-F., T. Rauscher, T., et al. 1999, ApJ, 516, 381

Goriely, S. 2000, in 10th Int. Symp. Capture gamma-ray spectroscopy and related topics (AIP), AIP Conf. Proc., 52, 287

Goriely, S., \& Clerbaux, B. 1999, A\&A, 346, 798

Goriely, S., Hilaire, S., \& Koning, A. J. 2008, A\&A., 487, 767

Goriely, S., Hilaire, S., Koning, A. J., Sin, M., \& Capote, R. 2009, Phys. Rev. C., 79, 024612, available online at http://www.astro.ulb.ac.be/Html

Goriely, S., \& Khan, E. 2002, Nucl. Phys. A, 706, 217

Hauser, W., \& Feshbach, H. 1952, Phys. Rev., 87, 366

Hilf, E. R., Groote, H. V., \& Takahashi, K. 1976, in Proc. 3rd Int. Conf. on Nuclei far from Stability, CERN-76-13 (CERN), 142

Holmes, J., Woosley, S., Fowler, W., \& Zimmerman, B. 1976, At. Data Nucl. Data Tables, 18, 305

Howard, W. M., \& Möller, P. 1980, At. Data Nucl. Data Tables, 25, 219

Kelić, A., Ricciardi, M. V., \& Schmidt, K.-H. 2008, in Joint ICTP-IAEA Advanced Workshop on Model Codes for Spallation Reactions, ed. D. F. et al., INDC(NDS)-530 (Vienna: IAEA), 181

Kelić, A., Zinner, N., Kolbe, E., Langanke, K., \& Schmidt, K.-H. 2005, Phys. Lett. B, 616, 48

Kodama, T., \& Takahashi, K. 1975, Nucl. Phys. A, 239, 489

Kruglov, K., Andreyev, A., Bruyneel, B. et al. 2002, Europ. Phys. J., A14, 365

Mamdouh, A., Pearson, J. M., Rayet, M., \& Tondeur, F. 1998, Nucl. Phys., A664, 389

Mamdouh, A., Pearson, J. M., Rayet, M., \& Tondeur, F. 2001, Nucl. Phys., A679, 337

Martínez-Pinedo, G., Mocelj, D., Zinner, N. T., et al. 2007, Prog. Part. Nucl. Phys., 59, 199

Möller, P., Nix, J. R., \& Kratz, K.-L. 1997, At. Data Nucl. Data Tables, 66, 131

Möller, P., Nix, J. R., Myers, W. D., \& Swiatecki, W. J. 1995, At. Data Nucl. Data Tables, 59, 185

Möller, P., Sierk, A. J., Ichikawa, T., et al. 2009, Phys. Rev. C., 79, 064304

Myers, W. D., \& Swiatecki, W. J. 1996, Nucl. Phys., A601, 141

Myers, W. D., \& Swiatecki, W. J. 1999, Phys. Rev. C, 60, 014606

Nadtochy, P.-N., Adeev, G.D., \& Karpo, A.V. 2005, Phys. Rev. C, 65, 064615

Nagy, S., Flynn, K. F., Gindler, J. E., Meadows, J. W., \& Glendenin, L. E. 1978, Phys. Rev. C, 17, 163

Nakagawa, T., Chiba, S., Hayakawa, T., \& Kajino, T. 2005, At. Data Nucl. Data Tables, 91, 77

Panov, I., \& Thielemann, F.-K. 2004, Astron. Lett., 30, 647

Panov, I. V., \& Thielemann, F.-K. 2003, Nucl. Phys., A718, 647

Panov, I. V., Kolbe, E., Pfeiffer, B., et al. 2005, Nucl. Phys., A747, 633

Panov, I. V., Korneev, I. Y., \& Thielemann, F.-K. 2008, Astron. Lett., 34, 189 Rauscher, T., \& Thielemann, F.-K. 2000, At. Data Nucl. Data Tables, 75, 1 Rauscher, T., \& Thielemann, F.-K. 2001, At. Data Nucl. Data Tables, 79, 47 Rauscher, T., Bieber, R., Oberhummer, H., et al. 1998, Phys. Rev. C, 57, 2031 
A\&A 513, A61 (2010)

Rauscher, T., Thielemann, F.-K., \& Kratz, K.-L. 1997, Phys. Rev. C, 56, 1613 Rauscher, T., Applegate, J., Cowan, J., Thielemann, F.-K., \& Wiescher, M. 1994, ApJ, 429, 499

Rusanov, A. Y., Itkis, M.G., \& Okolovich, V. N. 1997, Phys. At. Nucl., 60, 683 Sargood, D. G. 1982, Phys. Rep., 93, 61

Schmidt, K.-H., Kelić, A., \& Ricciardi, M. 2008, Europhys. Lett., 83, 32001

Smirenkin, G. N. 1993, Tech. Rep. INDC(CCP)-359, IAEA

Soppera, N., Bossant, M., Henriksson, H., Nagel, P., \& Rugama, Y. 2008, in Proc. Int. Conf. on Nuclear Data for Science and Technology, April 22-27, 2007, ed. e. a. O. Bersillon (EDP Sciences), 1

Strutinsky, V. M. 1967, Nucl. Phys., A95, 420

Thielemann, F.-K., Arnould, M., \& Truran, J. W. 1987, in Advances in Nuclear Astrophysics, ed. E. V.-F. et al. (Gif-sur-Yvette: Editions Frontières), 525
Thielemann, F.-K., Cameron, A., \& Cowan, J. J. 1989, in Fifty Years with Nuclear Fission, ed. J. Behrens \& A. Carlson (Gaithersburg: American Nuclear Society), 592

Thielemann, F.-K., Metzinger, J., \& Klapdor-Kleingrothaus, H. V. 1983, Z. Phys. A, 309, 301

Tovesson, F., Hill, T. S., Baker, J. D., \& McGrath, C. A. 2009, Phys. Rev. C., 79, 014613

Wahl, A. C. 1988, At. Data Nucl. Data Tables, 39, 1

Wilkins, B., Steinberg, E., \& Chasman, R. 1976, Phys. Rev. C, 14, 1832

Wolfenstein, L. 1951, Phys. Rev., 82, 690

Woosley, S., Fowler, W., Holmes, J., \& Zimmerman, B. 1978, At. Data Nucl. Data Tables, 22, 371 